\title{
Momentum flux determination using the multi-beam Poker Flat Incoherent Scatter Radar
}

\author{
M. J. Nicolls ${ }^{1}$, D. C. Fritts ${ }^{2}$, D. Janches ${ }^{3}$, and C. J. Heinselman ${ }^{1}$ \\ ${ }^{1}$ Center for Geospace Studies, SRI International, Menlo Park, CA, USA \\ ${ }^{2}$ Colorado Research Associates, NorthWest Research Associates, Boulder, CO, USA \\ ${ }^{3}$ NASA/Goddard Space Flight Center, Greenbelt, MD, USA
}

Correspondence to: M. J. Nicolls (michael.nicolls@sri.com)

Received: 3 January 2012 - Revised: 3 May 2012 - Accepted: 7 May 2012 - Published: 11 June 2012

\begin{abstract}
In this paper, we develop an estimator for the vertical flux of horizontal momentum with arbitrary beam pointing, applicable to the case of arbitrary but fixed beam pointing with systems such as the Poker Flat Incoherent Scatter Radar (PFISR). This method uses information from all available beams to resolve the variances of the wind field in addition to the vertical flux of both meridional and zonal momentum, targeted for high-frequency wave motions. The estimator utilises the full covariance of the distributed measurements, which provides a significant reduction in errors over the direct extension of previously developed techniques and allows for the calculation of an error covariance matrix of the estimated quantities. We find that for the PFISR experiment, we can construct an unbiased and robust estimator of the momentum flux if sufficient and proper beam orientations are chosen, which can in the future be optimized for the expected frequency distribution of momentumcontaining scales. However, there is a potential trade-off between biases and standard errors introduced with the new approach, which must be taken into account when assessing the momentum fluxes. We apply the estimator to PFISR measurements on 23 April 2008 and 21 December 2007, from 60-85 km altitude, and show expected results as compared to mean winds and in relation to the measured vertical velocity variances.
\end{abstract}

Keywords. Meteorology and atmospheric dynamics (Waves and tides; Instruments and techniques) - Radio science (Remote sensing)

\section{Introduction}

Geophysical vertical flux of horizontal momentum measurements were pioneered by Lhermitte (1983) and Vincent and Reid (1983), the former for studying the spectral variance in underwater tidal channels and the latter for assessing the vertical structure of wave motions in the atmosphere. In particular, Vincent and Reid (1983) developed a technique for ground-based radars to estimate radial velocity variances and corresponding momentum fluxes in the mesosphere and lower thermosphere (MLT) using dual co-planar narrow radar beams. This technique has since been widely used by HF and VHF radars (e.g., Reid and Vincent, 1987; Fritts and Vincent, 1987; Fukao et al., 1988; Reid et al., 1988; Fritts and Yuan, 1989; Fritts et al., 1990; Sato, 1990, 1993, 1994; Tsuda et al., 1990; Wang and Fritts, 1990, 1991; Hitchman et al., 1992; Nakamura et al., 1993; Murayama et al., 1994; Murphy and Vincent, 1993, 1998). Similar approaches have been extended to MF broad beam interferometric radars (Thorsen et al., 1997), incoherent scatter radars (ISRs) operating at VHF and UHF (Fritts et al., 1992; Fritts et al., 2006; Janches et al., 2006), and Doppler lidar systems (Acott, 2009). More recently, Hocking (2005) has generalized the approach for application to meteor radars, a technique that has been employed in several studies of gravity waves (GWs) (e.g., Antonita et al., 2008; Clemesha et al., 2009; Placke et al., 2011b,a), validated by Fritts et al. (2010) in applications of the SAAMER meteor radar in studies of mean winds, tides and GWs, and used by Vincent et al. (2010) in an assessment of GW momentum flux measurements by meteor radars. In this paper, we outline a technique for multi-beam radars to estimate the vertical flux of 
Table 1. Beam directions used for simulation and experiment.

\begin{tabular}{rrrrrr}
\hline & \multicolumn{2}{c}{ Beam map \#1 } & & \multicolumn{2}{c}{ Beam map \#2 } \\
\cline { 2 - 3 } \cline { 5 - 6 }$\#$ & Az. & El. & & Az. & El. \\
\hline 1 & N/A & $90.0^{\circ}$ & & N/A & $90.0^{\circ}$ \\
2 & $4.23^{\circ}$ & $73.73^{\circ}$ & & $0.0^{\circ}$ & $85.0^{\circ}$ \\
3 & $-1.81^{\circ}$ & $79.57^{\circ}$ & & $45.0^{\circ}$ & $85.0^{\circ}$ \\
4 & $82.04^{\circ}$ & $80.22^{\circ}$ & & $90.0^{\circ}$ & $85.0^{\circ}$ \\
5 & $91.92^{\circ}$ & $74.59^{\circ}$ & & $135.0^{\circ}$ & $85.0^{\circ}$ \\
6 & $-88.82^{\circ}$ & $80.73^{\circ}$ & & $180.0^{\circ}$ & $85.0^{\circ}$ \\
7 & $174.45^{\circ}$ & $81.43^{\circ}$ & & $-90.0^{\circ}$ & $85.0^{\circ}$ \\
\hline
\end{tabular}

horizontal momentum. While similar to the method of Hocking (2005), this technique is more applicable to the case of fixed-look directions determined a priori, rather than to the case of line-of-sight wind measurements from random meteor trail locations. This technique is particularly applicable to phased array radars such as the Advanced Modular Incoherent Scatter Radar (AMISR) class of radars.

\section{Momentum flux estimation - technique}

\subsection{Measurements}

ISRs are able to measure the line-of-sight (LOS) or radial ion/neutral velocity within a small volume in the MLT. A component velocity measurement $j$, made by an arbitrary beam, can be written as:

$v_{j}=\mathbf{a}_{j} \cdot \boldsymbol{v}+e_{j}$

where $\boldsymbol{v}=\left(v_{e}, v_{n}, v_{z}\right)$ is the vector velocity in geographic coordinates and $\boldsymbol{a}_{j}=\left(a_{j, e}, a_{j, n}, a_{j, z}\right)=$ $\left(\cos \theta_{j} \sin \phi_{j}, \cos \theta_{j} \cos \phi_{j}, \sin \theta_{j}\right)$ is a simplified straightline geometry vector with $\phi_{j}$ and $\theta_{j}$ being the azimuth (east of north) and elevation angles, respectively. (Note that throughout this paper, we will use the notation $a_{j, e}, a_{j, n}, a_{j, z}$ to refer to the eastward, northward, and vertical components for a given beam $j$.) The measurement has an associated error, $e_{j}$, with expected value $\left\langle e_{j}\right\rangle=0$.

Single instrument ISR experiments typically consist of measurements $v_{j}$ distributed in altitude, space and/or time depending on the system, its steering dexterity, etc. We will consider cases applicable to the pulse-to-pulse beam steering of the Poker Flat Incoherent Scatter Radar (PFISR).

\subsection{Vector velocities}

As described by Heinselman and Nicolls (2008) and Nicolls et al. (2010), an arbitrary number of component velocity measurements can be used to estimate the velocity vector.
This approach can be expressed in matrix form as

$$
\left[\begin{array}{c}
v_{1} \\
v_{2} \\
\vdots \\
v_{j}
\end{array}\right]=\left[\begin{array}{ccc}
a_{1, e} & a_{1, n} & a_{2, z} \\
a_{2, e} & a_{2, n} & a_{2, z} \\
\vdots & \vdots & \vdots \\
a_{j, e} & a_{j, n} & a_{j, z}
\end{array}\right]\left[\begin{array}{c}
v_{e} \\
v_{n} \\
v_{z}
\end{array}\right]+\left[\begin{array}{c}
e_{1} \\
e_{2} \\
\vdots \\
e_{j}
\end{array}\right]
$$

or

$\boldsymbol{v}_{\mathrm{los}}=\mathbf{A v}+\mathbf{e}$

A weighted linear least squares estimator for $v$ is then,

$\hat{\boldsymbol{v}}=\underset{\boldsymbol{v}}{\arg \min }\left(\left\|\boldsymbol{v}_{\mathrm{los}}-\mathbf{A} \boldsymbol{v}\right\|_{\mathbf{W}}^{2}\right)$

where $\|\cdot\|$ w represents a weighted $\ell^{2}$-norm with weight matrix given by $\mathbf{W}=\mathbf{C}^{-1}$, where $\mathbf{C}$ is the covariance matrix of the measurements. In this case, with uncorrelated errors, $\mathbf{C}$ is simply a diagonal matrix with diagonal elements given by $\left\langle e_{j}^{2}\right\rangle$, the estimated variances of the measurements, where the notation $\langle\cdot\rangle$ denotes an expected value, i.e.,

$\mathbf{C}=\left[\begin{array}{ccc}\left\langle e_{1}^{2}\right\rangle & 0 & 0 \\ \vdots & \ddots & \vdots \\ 0 & 0 & \left\langle e_{j}^{2}\right\rangle\end{array}\right]$

Solution to Eq. (4) is given by the normal equations (e.g., Aster et al., 2005; Tarantola, 2005),

$\left(\mathbf{A}^{T} \mathbf{C}^{-1} \mathbf{A}\right) \hat{\mathbf{v}}=\mathbf{A}^{T} \mathbf{C}^{-1} \boldsymbol{v}_{\text {los }}$

or, rearranging,

$\hat{\boldsymbol{v}}=\left(\mathbf{A}^{T} \mathbf{C}^{-1} \mathbf{A}\right)^{-1} \mathbf{A}^{T} \mathbf{C}^{-1} \boldsymbol{v}_{\text {los }}$.

This solution is equivalent to the maximum a posteriori (MAP) estimator. If $\mathbf{A}^{T} \mathbf{C}^{-1} \mathbf{A}$ is ill-conditioned, or if the application of a physical constraint is appropriate, a regularization/constraint term can be added. For example, this approach has been applied to this problem by Heinselman and Nicolls (2008) and Butler et al. (2010). The error covariance matrix of the solution without a constraint term is given by

$\mathbf{C}_{\hat{\mathbf{v}}}=\left(\mathbf{A}^{T} \mathbf{C}^{-1} \mathbf{A}\right)^{-1}$.

\subsection{Velocity variances and momentum fluxes}

An estimator for the radial velocity perturbation is

$\hat{v}_{j}^{\prime}=v_{j}-\left\langle v_{j}\right\rangle$

where we assume that after removal of the mean value, $\left\langle\hat{v}_{j}^{\prime}\right\rangle=0$. The variance of this quantity may be written as

$\left\langle\hat{v}_{j}^{\prime 2}\right\rangle=\left\langle v_{j}^{\prime 2}\right\rangle+\left\langle e_{j}^{2}\right\rangle$. 
This is a biased estimator (biased by $\left\langle e_{j}^{2}\right\rangle$ ) of the variance. Similarly, the covariance of any two measurements $(i \neq j)$ can be written as,

$$
\left\langle\hat{v}_{i}^{\prime} \hat{v}_{j}^{\prime}\right\rangle=\left\langle v_{i}{ }^{\prime} v_{j}{ }^{\prime}\right\rangle
$$

where measurement errors have been assumed to be uncorrelated.

The quantity $\left\langle v_{i}^{\prime} v_{j}^{\prime}\right\rangle$ may be related to component fluxes:

$$
\begin{aligned}
& \left\langle v_{i}^{\prime} v_{j}^{\prime}\right\rangle=\left\langle v_{n}^{\prime 2}\right\rangle a_{i, n} a_{j, n}+\left\langle v_{e}^{\prime 2}\right\rangle a_{i, e} a_{j, e}+\left\langle v_{z}^{\prime 2}\right\rangle a_{i, z} a_{j, z} \\
& +\left\langle v_{n}^{\prime} v_{e}^{\prime}\right\rangle\left(a_{i, e} a_{j, n}+a_{j, e} a_{i, n}\right)+\left\langle v_{n}^{\prime} v_{z}^{\prime}\right\rangle\left(a_{i, n} a_{j, z}+a_{j, n} a_{i, z}\right) \\
& +\left\langle v_{e}^{\prime} v_{z}^{\prime}\right\rangle\left(a_{i, e} a_{j, z}+a_{j, e} a_{i, z}\right) .
\end{aligned}
$$

For the case of $i=j$ (variance measurement),

$$
\begin{aligned}
\left\langle v_{j}{ }^{\prime 2}\right\rangle= & \left\langle v_{n}^{\prime 2}\right\rangle a_{j, n}^{2}+\left\langle v_{e}^{\prime 2}\right\rangle a_{j, e}^{2}+\left\langle v_{z}^{\prime 2}\right\rangle a_{j, z}^{2}+ \\
& 2\left\langle v_{n}^{\prime} v_{e}^{\prime}\right\rangle a_{j e} a_{j, n}+2\left\langle v_{n}^{\prime} v_{z}^{\prime}\right\rangle a_{j, n} a_{j, z}+2\left\langle v_{e}^{\prime} v_{z}^{\prime}\right\rangle a_{j, e} a_{j, z} .
\end{aligned}
$$

The vertical fluxes of horizontal momentum correspond to the two last terms, $\left\langle v_{n}^{\prime} v_{z}^{\prime}\right\rangle$ and $\left\langle v_{e}^{\prime} v_{z}^{\prime}\right\rangle$, which we would like to estimate. Several techniques exist in estimating these quantities, including choosing look directions such that unwanted terms in Eq. (13) cancel (such as the co-planar beam method of Vincent and Reid (1983)) or by formulating a matrix of scaled variances, applicable to arbitrary pointing/meteor trail drift measurements (Hocking, 2005). Note that, throughout this section, it is implicitly assumed that the time-averaged quantities are constant over the probing volume - an assumption of horizontal homogeneity. This is assumed to be true of the background wind fields (driven by tidal and other lowfrequency motions) as well as the amplitudes of the wave fields. However, covariance measurements involve the estimation of the expected value of non-homogenous quantities (i.e., $v_{i}^{\prime}$, the velocity perturbation of a single measurement); thus, one must be cautious when including these terms using distributed measurements. This will be discussed in more detail in the simulation of realistic wave fields (Sect. 3.3).

More generally, if the full covariance matrix of measurements is able to be formed, then this can be related to the covariance matrix of the wind field by a standard covariance matrix transformation,

$$
\boldsymbol{\Sigma}=\mathbf{A D A}^{T} \text {. }
$$

Here, for a number of measurements $N_{\text {meas }}$ (corresponding, for example, to the number of look directions or beams), $\boldsymbol{\Sigma}$ is the $N_{\text {meas }} \times N_{\text {meas }}$ covariance matrix of the measurements,

$\boldsymbol{\Sigma}=\left[\begin{array}{cccc}\left\langle\hat{v}_{1}^{\prime 2}\right\rangle & \left\langle\hat{v}_{1}^{\prime} \hat{v}_{2}^{\prime}\right\rangle & \ldots & \left\langle\hat{v}_{1}^{\prime} \hat{v}_{j}^{\prime}\right\rangle \\ \left\langle\hat{v}_{1}^{\prime} \hat{v}_{2}^{\prime}\right\rangle & \left\langle\hat{v}_{2}^{\prime 2}\right\rangle & \ldots & \left\langle\hat{v}_{2}^{\prime} \hat{v}_{j}^{\prime}\right\rangle \\ \vdots & \vdots & \ddots & \vdots \\ \left\langle\hat{v}_{1}^{\prime} \hat{v}_{j}^{\prime}\right\rangle & \left\langle\hat{v}_{2}^{\prime} \hat{v}_{j}^{\prime}\right\rangle & \ldots & \left\langle\hat{v}_{j}^{\prime 2}\right\rangle\end{array}\right]$

the observation matrix A has been defined by Eq. (2), and the wind covariance matrix is given by

$\mathbf{D}=\left[\begin{array}{ccc}\left\langle v_{e}^{2}\right\rangle & \left\langle v_{e} v_{n}\right\rangle & \left\langle v_{e} v_{z}\right\rangle \\ \left\langle v_{e} v_{n}\right\rangle & \left\langle v_{n}^{2}\right\rangle & \left\langle v_{n} v_{z}\right\rangle \\ \left\langle v_{e} v_{z}\right\rangle & \left\langle v_{n} v_{z}\right\rangle & \left\langle v_{z}^{2}\right\rangle\end{array}\right]$.

A solution to Eq. (14) can be found directly,

$$
\hat{\mathbf{D}}=\left(\mathbf{A}^{T} \mathbf{A}\right)^{-1} \mathbf{A}^{T} \mathbf{\Sigma} \mathbf{A}\left(\mathbf{A}^{T} \mathbf{A}\right)^{-1} .
$$

While useful, this approach is more readily solvable by transforming the two-dimensional $\boldsymbol{\Sigma}$ and $\mathbf{D}$ matrices into onedimensional matrices of observational and estimated quantities. For simplicity and convenience, in our case we simply solve a linear least squares problem for 6 unknowns, $\left\langle v_{n}^{\prime 2}\right\rangle$, $\left\langle v_{e}^{\prime 2}\right\rangle,\left\langle v_{z}^{\prime 2}\right\rangle,\left\langle v_{n}^{\prime} v_{e}^{\prime}\right\rangle,\left\langle v_{n}^{\prime} v_{z}^{\prime}\right\rangle,\left\langle v_{e}^{\prime} v_{z}^{\prime}\right\rangle$. In this case, we may write,

$$
\boldsymbol{\Sigma}_{v}=\left[\begin{array}{c}
\left\langle\hat{v}_{1}^{\prime 2}\right\rangle \\
\left\langle\hat{v}_{1}^{\prime} \hat{v}_{2}^{\prime}\right\rangle \\
\vdots \\
\left\langle\hat{v}_{1}^{\prime} \hat{v}_{j}^{\prime}\right\rangle \\
\left\langle\hat{v}_{2}^{\prime 2}\right\rangle \\
\left\langle\hat{v}_{2}^{\prime} \hat{v}_{3}^{\prime}\right\rangle \\
\vdots \\
\left\langle\hat{v}_{2}^{\prime} \hat{v}_{j}^{\prime}\right\rangle \\
\vdots \\
\left\langle\hat{v}_{j}^{\prime 2}\right\rangle
\end{array}\right]=\mathbf{B}\left[\begin{array}{c}
\left\langle v_{e}^{2}\right\rangle \\
\left\langle v_{n}^{2}\right\rangle \\
\left\langle v_{z}^{2}\right\rangle \\
\left\langle v_{e} v_{n}\right\rangle \\
\left\langle v_{e} v_{z}\right\rangle \\
\left\langle v_{n} v_{z}\right\rangle
\end{array}\right]=\mathbf{B} \boldsymbol{D}_{v}
$$

defining $\boldsymbol{D}_{v}$ to be the vector of variances and co-variances. For $N_{\text {meas }}$ measurements/beams/look directions, the observation matrix $\boldsymbol{\Sigma}_{v}$ will be size $N_{\text {meas }}\left(N_{\text {meas }}+1\right) / 2$. The geometry matrix B is size $N_{\text {meas }}\left(N_{\text {meas }}+1\right) / 2 \times 6$, with elements given by the factors in Eq. (12), 
$\mathbf{B}=\left[\begin{array}{c|c|c|c|c|c}a_{1, e}^{2} & a_{1, n}^{2} & a_{1, z}^{2} & 2 a_{1, e} a_{1, n} & 2 a_{1, e} a_{1, z} & 2 a_{1, n} a_{1, z} \\ a_{1, e} a_{2, e} & a_{1, n} a_{2, n} & a_{1, z} a_{2, z} & a_{1, e} a_{2, n}+a_{2, e} a_{1, n} & a_{1, e} a_{2, z}+a_{2, e} a_{1, z} & a_{1, n} a_{2, z}+a_{2, n} a_{1, z} \\ \vdots & \vdots & \vdots & \vdots & \vdots & \vdots \\ a_{1, e} a_{j, e} & a_{1, n} a_{j, n} & a_{1, z} a_{j, z} & a_{1, e} a_{j, n}+a_{j, e} a_{1, n} & a_{1, e} a_{j, z}+a_{j, e} a_{1, z} & a_{1, n} a_{j, z}+a_{j, n} a_{1, z} \\ a_{2, e}^{2} & a_{2, n}^{2} & a_{2, z}^{2} & 2 a_{2, e} a_{2, n} & 2 a_{2, e} a_{2, z} & 2 a_{2, n} a_{2, z} \\ a_{2, e} a_{3, e} & a_{2, n} a_{3, n} & a_{2, z} a_{3, z} & a_{2, e} a_{3, n}+a_{3, e} a_{2, n} & a_{2, e} a_{3, z}+a_{3, e} a_{2, z} & a_{2, n} a_{3, z}+a_{3, n} a_{2, z} \\ \vdots & \vdots & \vdots & \vdots & \vdots & \vdots \\ a_{2, e} a_{j, e} & a_{2, n} a_{j, n} & a_{2, z} a_{j, z} & a_{2, e} a_{j, n}+a_{j, e} a_{2, n} & a_{2, e} a_{j, z}+a_{j, e} a_{2, z} & a_{2, n} a_{j, z}+a_{j, n} a_{2, z} \\ \vdots & \vdots & \vdots & \vdots & \vdots & \vdots \\ a_{j, e}^{2} & a_{j, n}^{2} & a_{j, z}^{2} & 2 a_{j, e} a_{j, n} & 2 a_{j, e} a_{j, z} & 2 a_{j, n} a_{j, z}\end{array}\right]$.

The inclusion of the covariance terms is a unique aspect of this approach and distinguishes it from previous techniques. If those terms were unable to be estimated or were ignored, than the approach would reduce to that of Hocking (2005), except that Hocking (2005) formed vectors of weighted variances and a corresponding geometry matrix, such that the system is exactly determined. That approach seems appropriate for meteor scattering for arbitrary locations and times over a large field-of-view. In the way we have formulated it, the problem may be over- or underdetermined and is an appropriate formulation for fixed experimental beam pointing, wherein the $\mathbf{B}$ matrix will remain fixed for any given experiment and where the covariance matrix of the measurements can be computed. We will show via simulation that including the covariance terms reduces the standard errors significantly in the presence of non-zero measurement uncertainties.

The estimator using the same approach as in the previous section is

$\hat{\mathbf{D}}=\left(\mathbf{B}^{T} \Sigma_{v v}^{-1} \mathbf{B}\right)^{-1} \mathbf{B}^{T} \Sigma_{v v}^{-1} \boldsymbol{\Sigma}_{v}$.

A great advantage of this approach is that the a posteriori error covariance matrix (errors on the wind variances and momentum fluxes) can be readily estimated. This matrix is given by

$\Sigma_{\hat{D}_{v}}=\left(\mathbf{B} \Sigma_{v v}^{-1} \mathbf{B}^{T}\right)^{-1}$.

Here, $\Sigma_{v v}$ is the covariance matrix of the measurements $\boldsymbol{\Sigma}_{v}$, which can be readily computed. The general covariance of any entry in the covariance matrix is given by,

$$
\begin{aligned}
\operatorname{Cov}\left(\hat{v}_{i}^{\prime} \hat{v}_{j}^{\prime}, \hat{v}_{m}^{\prime} \hat{v}_{n}^{\prime}\right)= & \left\langle\hat{v}_{i}^{\prime} \hat{v}_{j}^{\prime} \hat{v}_{m}^{\prime} \hat{v}_{n}^{\prime}\right\rangle-\left\langle\hat{v}_{i}^{\prime} \hat{v}_{j}^{\prime}\right\rangle\left\langle\hat{v}_{m}^{\prime} \hat{v}_{n}^{\prime}\right\rangle \\
= & \left\langle\hat{v}_{i}^{\prime} \hat{v}_{m}^{\prime}\right\rangle\left\langle\hat{v}_{j}^{\prime} \hat{v}_{n}^{\prime}\right\rangle+\left\langle\hat{v}_{i}^{\prime} \hat{v}_{n}^{\prime}\right\rangle\left\langle\hat{v}_{j}^{\prime} \hat{v}_{m}^{\prime}\right\rangle \\
& +\left\langle\hat{v}_{i}^{\prime} \hat{v}_{m}^{\prime}\right\rangle\left\langle e_{j, n}^{2}\right\rangle \delta_{j, n}+\left\langle\hat{v}_{i}^{\prime} \hat{v}_{n}^{\prime}\right\rangle\left\langle e_{j, m}^{2}\right\rangle \delta_{j, m} \\
& +\left\langle\hat{v}_{j}^{\prime} \hat{v}_{m}^{\prime}\right\rangle\left\langle e_{i, n}^{2}\right\rangle \delta_{i, n}+\left\langle\hat{v}_{j}^{\prime} \hat{v}_{n}^{\prime}\right\rangle\left\langle e_{i, m}^{2}\right\rangle \delta_{i, m} \\
& +\left\langle e_{i, m}^{2}\right\rangle\left\langle e_{j, n}^{2}\right\rangle \delta_{i, m} \delta_{j, n}+\left\langle e_{i, n}^{2}\right\rangle\left\langle e_{j, m}^{2}\right\rangle \delta_{i, n} \delta_{j, m}
\end{aligned}
$$

where we have used the fourth moment theorem for multivariate normally distributed variables to expand the higher order moments, and $\delta_{i, j}$ is the Kronecker delta ( 1 for $i=j$, 0 otherwise). For example, we see that if $i=j=m=n$, then

$\operatorname{Cov}\left(\hat{v}_{i}^{\prime 2}, \hat{v}_{i}^{\prime 2}\right)=2\left(\left\langle v_{i}^{\prime 2}\right\rangle+\left\langle e_{i}^{2}\right\rangle\right)^{2}$

For $(i=j) \neq(m=n)$,

$\operatorname{Cov}\left(\hat{v}_{i}^{\prime 2}, \hat{v}_{j}^{\prime 2}\right)=2\left\langle v_{i}^{\prime} v_{j}^{\prime}\right\rangle^{2}$

For $(i=m) \neq(j=n)$,

$\operatorname{Cov}\left(\hat{v}_{i}^{\prime} \hat{v}_{j}^{\prime}, \hat{v}_{i}^{\prime} \hat{v}_{j}^{\prime}\right)=\left(\left\langle v_{i}{ }^{\prime 2}\right\rangle+\left\langle e_{i}^{2}\right\rangle\right)\left(\left\langle v_{j}{ }^{2}\right\rangle+\left\langle e_{j}^{2}\right\rangle\right)+\left\langle v_{i}^{\prime} v_{j}{ }^{\prime}\right\rangle^{2}$

For $i \neq j \neq m \neq n$,

$\operatorname{Cov}\left(\hat{v}_{i}^{\prime} \hat{v}_{j}^{\prime}, \hat{v}_{m}^{\prime} \hat{v}_{n}^{\prime}\right)=\left\langle v_{i} v_{m}\right\rangle\left\langle v_{j} v_{n}\right\rangle+\left\langle v_{i} v_{n}\right\rangle\left\langle v_{j} v_{m}\right\rangle ;$

and for $i=m, j \neq n$,

$\operatorname{Cov}\left(\hat{v}_{i}^{\prime} \hat{v}_{j}^{\prime}, \hat{v}_{i}^{\prime} \hat{v}_{n}^{\prime}\right)=\left(\left\langle v_{i}^{\prime 2}\right\rangle+\left\langle e_{i}^{2}\right\rangle\right)\left\langle v_{j} v_{n}\right\rangle+\left\langle v_{i} v_{n}\right\rangle\left\langle v_{i} v_{j}\right\rangle$

From these expressions, the full covariance matrix of the measurements can be computed, which can be propagated to compute the variance of the estimator. Misestimation of the covariance matrix and violation of the linear least squares assumptions of normality can lead to biased estimates of the momentum fluxes. As a result, in practice, it may be better to use a diagonal prior covariance matrix and only use the estimated covariance matrix for estimates of the errors on the results.

As a final note, the bias in Eq. (10) can cause biases in the estimated quantities, particularly if the covariance terms are included as described here. While the biases on the variance estimates can be removed if they are known, we have found that it may be better to estimate them as well. The approach described above can be augmented to account for the biases. In this approach, the forward model is more correctly 

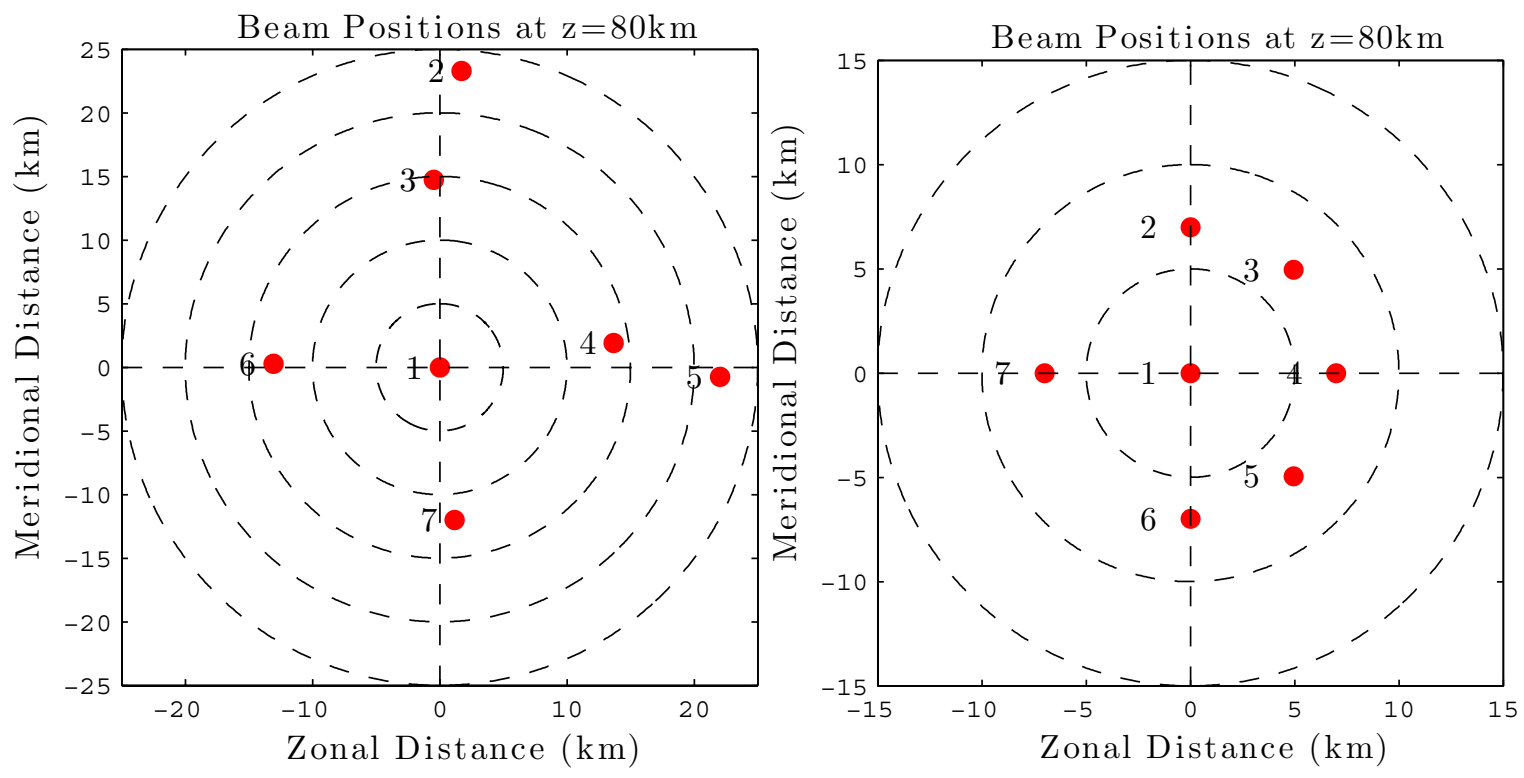

Fig. 1. PFISR beam position maps used in this paper, each with 7 look directions and evaluated at $80 \mathrm{~km}$ altitude. Left: A standard experimental beam map that has been used in several PFISR experiments. Right: A theoretical beam map with smaller off-zenith angles. Azimuth and elevation angles are listed in Table 1.

described to include the bias terms and Eq. (14) is modified such that

$\boldsymbol{\Sigma}=\mathbf{A D A}^{T}+\mathbf{C}$

where $\mathbf{C}$ is the diagonal measurement error covariance matrix given by Eq. (5). Equation (18) can then also be modified to add $N_{\text {meas }}$ additional unknowns $\left(\left\langle e_{1}^{2}\right\rangle,\left\langle e_{2}^{2}\right\rangle \ldots\right)$ with a corresponding modification in the $\mathbf{B}$ matrix.

\section{Momentum flux estimation - simulation}

\subsection{Simulation description}

In this section, we use the principles developed in Sect. 2 to perform a simulation of momentum flux extraction. For these simulations, we use two experiment geometries, as indicated in Table 1 and plotted at $80 \mathrm{~km}$ altitude in Fig. 1. Beam map \#1 is the experiment geometry used by the Dregion PFISR measurements of Nicolls et al. (2010). This experiment consists of seven look directions, which are listed and numbered in Table 1, and include a vertical look direction. Beam map \#2 is a theoretical beam map with the same number of positions (7), but with the maximum off-zenith angle reduced to $5^{\circ}$, for reasons that will become apparent in the simulation of realistic wave fields.

Given these beam directions, we can directly compute the a posteriori covariance matrix of the estimator using Eq. (21), assuming a diagonal a priori covariance matrix (in general, this is not a good assumption, as the a priori covariance matrix can be highly non-diagonal given both the properties of the wind field and the fact that measurements are reused to compute the full covariance matrix; this exercise is merely to show the influence of the observation matrix). In Fig. 2, we utilise this calculation to investigate the importance of each beam from Table 1 (beam map \#1) in the estimation procedure. In the future, a similar procedure could be used to optimize experiment pointing geometry. The output error matrix of the estimated wind covariances is estimated after removing each beam sequentially from the estimation procedure. The fractional increase in the variance of each estimated quantity is indicated in Fig. 2 (black dots). The red dots show the same calculation, but for the procedure neglecting the measurement covariance terms that were included in the estimator in Sect. 2.

In general, we see that $\left\langle v_{e}^{2}\right\rangle$ and $\left\langle v_{n}^{2}\right\rangle$ are most sensitive to the removal of beams $(5,6)$ and $(2,7)$, respectively. This is not surprising, as these beams have the lowest elevation angles and are the most non-redundant look directions. The quantity $\left\langle v_{z}^{2}\right\rangle$ is very well determined and not that sensitive to any beams. This is somewhat surprising, given the presence of a vertical beam, but the fact that all beams have fairly high elevation angles means that they are all sensitive to vertical motions (and, note that this calculation does not take into account anything about the magnitude of the component variances, expected to be much smaller in the vertical direction). The horizontal wind covariance term, $\left\langle v_{e} v_{n}\right\rangle$, is the most poorly determined parameter and roughly equally sensitive to all beams other than the vertical beam. Note that the chosen experiment geometry pointed beams in roughly cardinal directions, which could be improved/optimized in future experiments with consideration about sensitivity to the dominant 

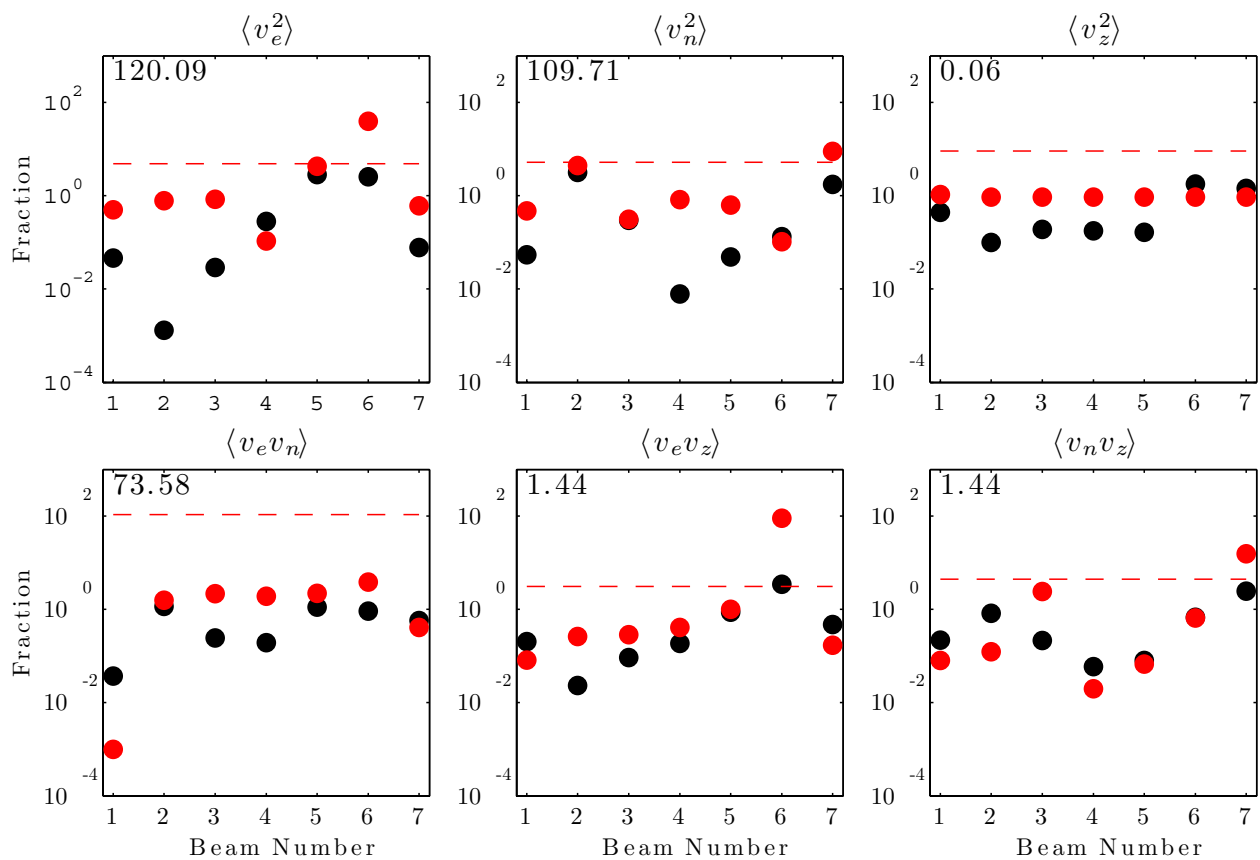

Fig. 2. Using beam map \#1, estimate of the fractional increase in the variance of each estimated wind variance/covariance term after removing individual beams (indicated by the $\mathrm{x}$-axis) from the estimation procedure, for (black) procedure including covariance terms and (red) procedure neglecting covariance terms. This process assumes a diagonal measurement error covariance matrix. The absolute value of the variance (for a measurement error covariance matrix given by the identity matrix) using all beams is indicated in the upper left of each panel for the procedure including the covariance terms. The ratio of the absolute values using all beams is indicated by the horizontal red dashed line. This turns out to be an overestimate, due mostly to the covariance of the measurements.

scales and frequencies. The momentum flux terms, $\left\langle v_{e} v_{z}\right\rangle$ and $\left\langle v_{n} v_{z}\right\rangle$ seem fairly well-determined and largely sensitive to individual beams pointed in the westward and southward directions, respectively, again due to the non-redundancy of these beams.

Comparing the results, including the covariance terms outlined in Sect. 2 (black) and neglecting those terms (red), we see that in general including the covariance terms leads to less sensitivity in removing individual beams. The absolute magnitude of the variances is much smaller for the estimates including the covariances - a factor of $\sim 10$ in root-meansquare error for the term $\left\langle v_{e} v_{n}\right\rangle$, and a factor of $\sim 1.5-3$ for all other terms. However, these estimates neglect the role of nondiagonal measurement error covariance matrix, which will be included next when examining the results of simulations.

To investigate the statistical properties and any biases associated with momentum flux extraction using the technique developed, we performed two types of simulations: the first (Sect. 3.2), to investigate the statistical aspects of momentum flux extraction, and the second (Sect. 3.3) to simulate realistic wave fields.

\subsection{Simulation 1: statistical properties of momentum flux extraction}

For the purposes of an initial simulation designed to investigate the statistical properties of momentum flux extraction, we use the beam configuration described in the previous section with an assumed, non-essential background wind environment $\overline{\boldsymbol{v}}$. We then perform the following steps for $N_{1}$ iterations of the simulation:

- Generate a $3 \times 3$ matrix, $\mathbf{L}$, with elements drawn from a uniform distribution from -5 to $5 \mathrm{~m} \mathrm{~s}^{-1}$.

- Use this matrix to generate a symmetric positivesemidefinite covariance matrix $\mathbf{L}^{T} \mathbf{L}$ which now represents the wind variances $\left(\left\langle v_{e}^{2}\right\rangle,\left\langle v_{n}^{2}\right\rangle\right.$ and $\left\langle v_{z}^{2}\right\rangle$, diagonal elements) and covariances $\left(\left\langle v_{e} v_{n}\right\rangle,\left\langle v_{e} v_{z}\right\rangle\right.$ and $\left\langle v_{n} v_{z}\right\rangle$, off-diagonal elements). This approach will generate a wind covariance matrix with variance terms with a median value of $\sim 25 \mathrm{~m}^{2} \mathrm{~s}^{-2}$ and ranging up to $\sim 50$ $70 \mathrm{~m}^{2} \mathrm{~s}^{-2}$. Covariance terms will have a median value close to $0 \mathrm{~m}^{2} \mathrm{~s}^{-2}$, ranging from $\sim-50$ to $50 \mathrm{~m}^{2} \mathrm{~s}^{-2}$.

- For $N_{2}$ iterations, generate a time series of wind vectors with length $N_{3}$ points (corresponding to the number of samples used to estimate the variances, covariances and wind vectors), drawn from the multi-variate normal 

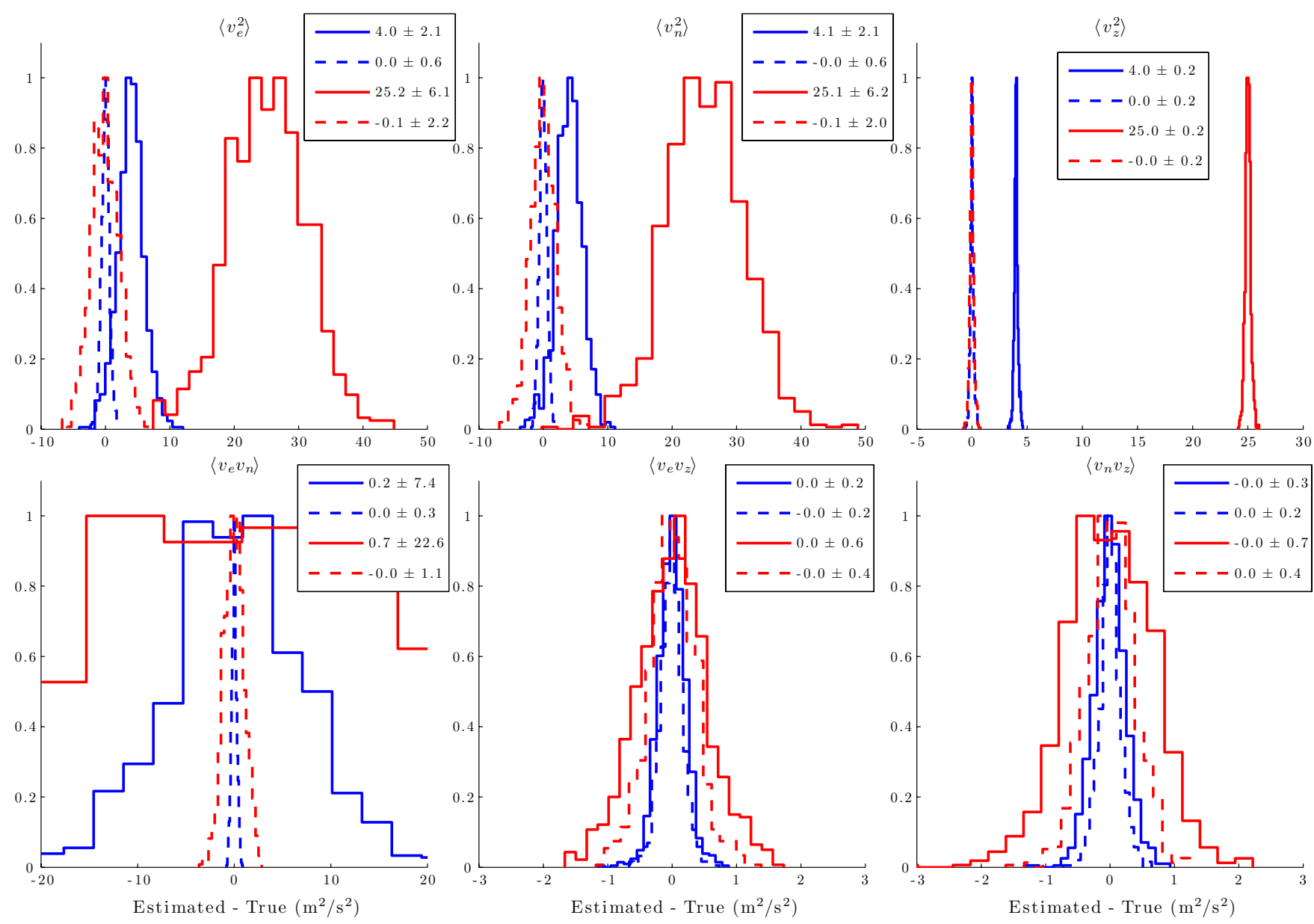

Fig. 3. Simulation results. Histograms of the biases (true value subtracted from the mean result of the simulation over $N_{2}$ iterations) for each wind field variance/covariance measurement. Blue lines correspond to $\sigma_{e}=2 \mathrm{~m} \mathrm{~s}^{-1}$ and red lines correspond to $\sigma_{e}=5 \mathrm{~m} \mathrm{~s}{ }^{-1}$. Solid lines correspond to the standard momentum flux approach, neglecting the covariance terms. Dashed lines follow the approach outlined in this paper including the covariance terms and subtracting the expected value of the measurement error variances from the estimated variances. Legend values show the median and standard deviation of each dataset.

distribution with covariance matrix $\mathbf{L}^{T} \mathbf{L}$ and mean $\overline{\boldsymbol{v}}$. Note that the resulting uncertainties on the wind variances and covariances will be highly dependent on $N_{3}$, as shown and discussed later.

- For each look direction, generate observed line-of-sight wind components using Eq. (9) with errors drawn from the zero-mean normal distribution with variance $\left\langle e^{2}\right\rangle$.

- Utilise Eq. (20) to estimate the momentum flux and wind component variances, as well as estimated measurement errors $\left\langle\hat{e}_{j}^{2}\right\rangle$.

- Estimate the means and standard deviations of the variance and covariance terms over the $N_{2}$ iterations, which can be used to compute the standard error on the estimate as well as any biases on the results.

We have run simulations as described above using $N_{1}=$ $N_{2}=1000$ and $N_{3}=60$, with $\sigma_{e}=\sqrt{\left\langle e^{2}\right\rangle}$ equal to 0,2 and
$5 \mathrm{~m} \mathrm{~s}^{-1}$. Results are shown in Fig. 3, where each panel shows a histogram of the difference between the true value and the estimated value (averaged over the $N_{2}$ iterations). This represents a histogram of the bias of the estimator. Here, blue lines correspond to the $\sigma_{e}=2 \mathrm{~m} \mathrm{~s}^{-1}$ case and red lines correspond to the $\sigma_{e}=5 \mathrm{~m} \mathrm{~s}^{-1}$ case. Solid lines correspond to the standard momentum flux approach, neglecting the covariance terms, whereas dashed lines correspond to the technique described in this paper.

The variances, $\left\langle v_{e}^{2}\right\rangle,\left\langle v_{n}^{2}\right\rangle$ and $\left\langle v_{z}^{2}\right\rangle$, are reasonably well determined for the small error case, especially in the vertical direction. As already mentioned, this is due to the high elevation angles of the beams and the presence of a vertical beam. However, the variances are clearly biased (in all three directions), which is most clearly seen on examination of the $\left\langle v_{z}^{2}\right\rangle$ histogram where the red curves are offset by $25 \mathrm{~m}^{2} \mathrm{~s}^{-2}$ (and the blue curves by $4 \mathrm{~m}^{2} \mathrm{~s}^{-2}$ ). As discussed in Sect. 2, there is an expected bias in the line-of-sight variance by $\left\langle e^{2}\right\rangle$ 

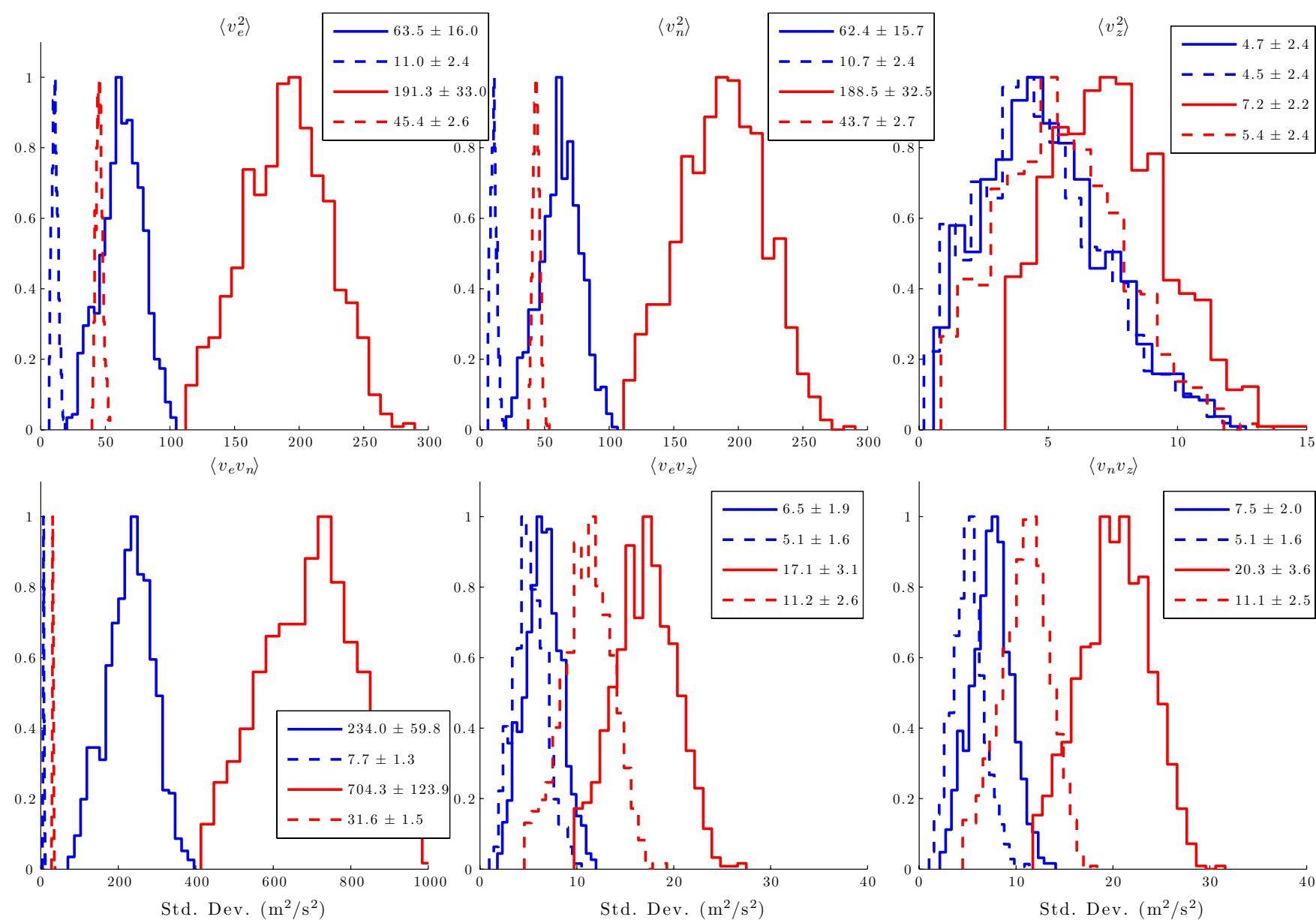

Fig. 4. Similar to Fig. 3, except curves correspond to histograms of standard deviations of wind variance and momentum flux estimates, computed over the $\mathrm{N}_{2}$ iterations.

that propagates to the wind variances. This bias is removed in the approach described (dashed lines), because an estimate of the measurement error variance was removed from the measurement variances. The bias, however, does not affect the covariance terms. For these terms, we see unbiased results in all cases, relatively poorly determined for the $\left\langle v_{e} v_{n}\right\rangle$ term and reasonably well determined for the vertical flux results.

The approach using the covariance terms (dashed lines) significantly outperforms the traditional approach, as seen by the standard deviation of the biases - the distribution of the biases is much narrower. This is especially true for the $\left\langle v_{e} v_{n}\right\rangle$ term, which is due to the additional information provided by the covariances.

The errors in the approach are quantified in more detail in Fig. 4, where we plot histograms of the standard deviations of the estimates in a similar format to Fig. 3. Standard deviations were computed using the $N_{2}$ iterations and represent the error in the estimate. Note that we earlier described an approach to estimate errors from the covariance matrix of the measurements, and we have confirmed that the standard deviations plotted in Fig. 4 agree with these esti- mates. Errors increase significantly as $\sigma_{e}$ increases, but are much lower, for all parameters, for the technique using the covariance terms. For the momentum flux terms, the errors decrease by about $25 \%$ for the $\sigma_{e}=2 \mathrm{~m} \mathrm{~s}^{-1}$ case and about $40 \%$ for the $\sigma_{e}=5 \mathrm{~m} \mathrm{~s}^{-1}$.

The conclusion that we draw from these simulations is that with (1) appropriate beam geometry and (2) sufficiently precise measurements, we can make unbiased estimates with reasonable errors of the vertical flux of horizontal momentum. These errors can quickly become untenable if these criteria are relaxed. Errors are significantly reduced if the full covariance matrix of the measurements can be computed, as is the case for PFISR measurements.

Given that the momentum flux is a measure of the correlation between horizontal and vertical wind variations, its determination is statistical in nature: a single measurement, even with no instrument errors, has at least $100 \%$ uncertainties, determined by the geometric mean of the horizontal and vertical wind variances (e.g., $\sqrt{\left\langle v_{n}^{2}\right\rangle\left\langle v_{z}^{2}\right\rangle}$ ) and the fractional variance embodied in the momentum flux term (e.g., 

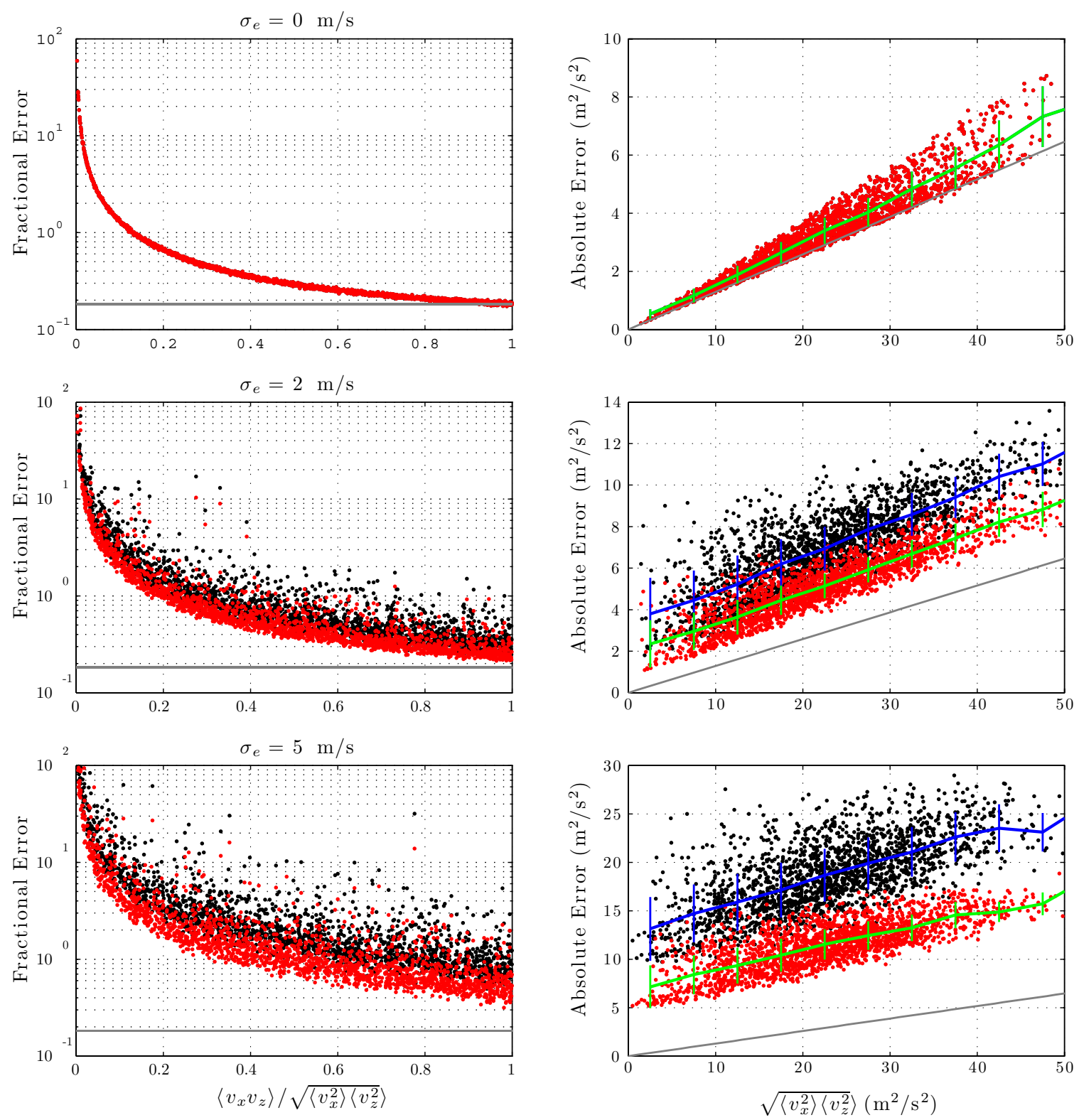

Fig. 5. The top row corresponds to $\sigma_{e}=0 \mathrm{~m} \mathrm{~s}^{-1}$, the middle row to $\sigma_{e}=2 \mathrm{~m} \mathrm{~s}^{-1}$, and the bottom row to $\sigma_{e}=5 \mathrm{~m} \mathrm{~s}^{-1}$. Left: Fractional error in horizontal momentum flux determination as a function of horizontal to vertical wind correlation. As the correlation approaches 1 , the fractional error approaches $\sqrt{2} / \sqrt{N_{3}}=0.18$ (solid horizontal line). Right: Absolute error in horizontal momentum flux as a function of $\sqrt{\left\langle v_{x}^{2}\right\rangle\left\langle v_{z}^{2}\right\rangle}$ (where results for $\left\langle v_{e} v_{z}\right\rangle$ and $\left\langle v_{n} v_{z}\right\rangle$ have been merged). Blue and green lines with errorbars show mean and $1 \sigma$ spread for the black and red points, respectively, and gray line corresponds to $\sqrt{\left\langle v_{x}^{2}\right\rangle\left\langle v_{z}^{2}\right\rangle / N_{3}}$. In all panels, black dots are from the simulation neglecting the covariance terms, where red dots are from the approach described in the text.

Kudeki and Franke, 1998; Thorsen et al., 2000; Vincent et al., 2010). For the case of this simulation, we have assumed that $N_{3}=60$ statistically independent points exist with which to compute the line-of-sight variances and corresponding wind variances and co-variances. In Fig. 5, this issue is demonstrated.

Figure 5 (left) shows the fractional error in estimating the horizontal momentum flux as a function of the correlation between the horizontal and vertical wind components, for the case of no measurement errors. In this case, the approach described here including the covariance terms agrees exactly with the traditional approach (or, that of Hocking, 2005, for arbitrary pointing). When the correlation is near 1 , the fractional error approaches $\sqrt{2} / \sqrt{N_{3}}=0.18$. As the correlation decreases, the fractional error increases dramatically, demonstrating the importance of this quantity (e.g., see Kudeki and 

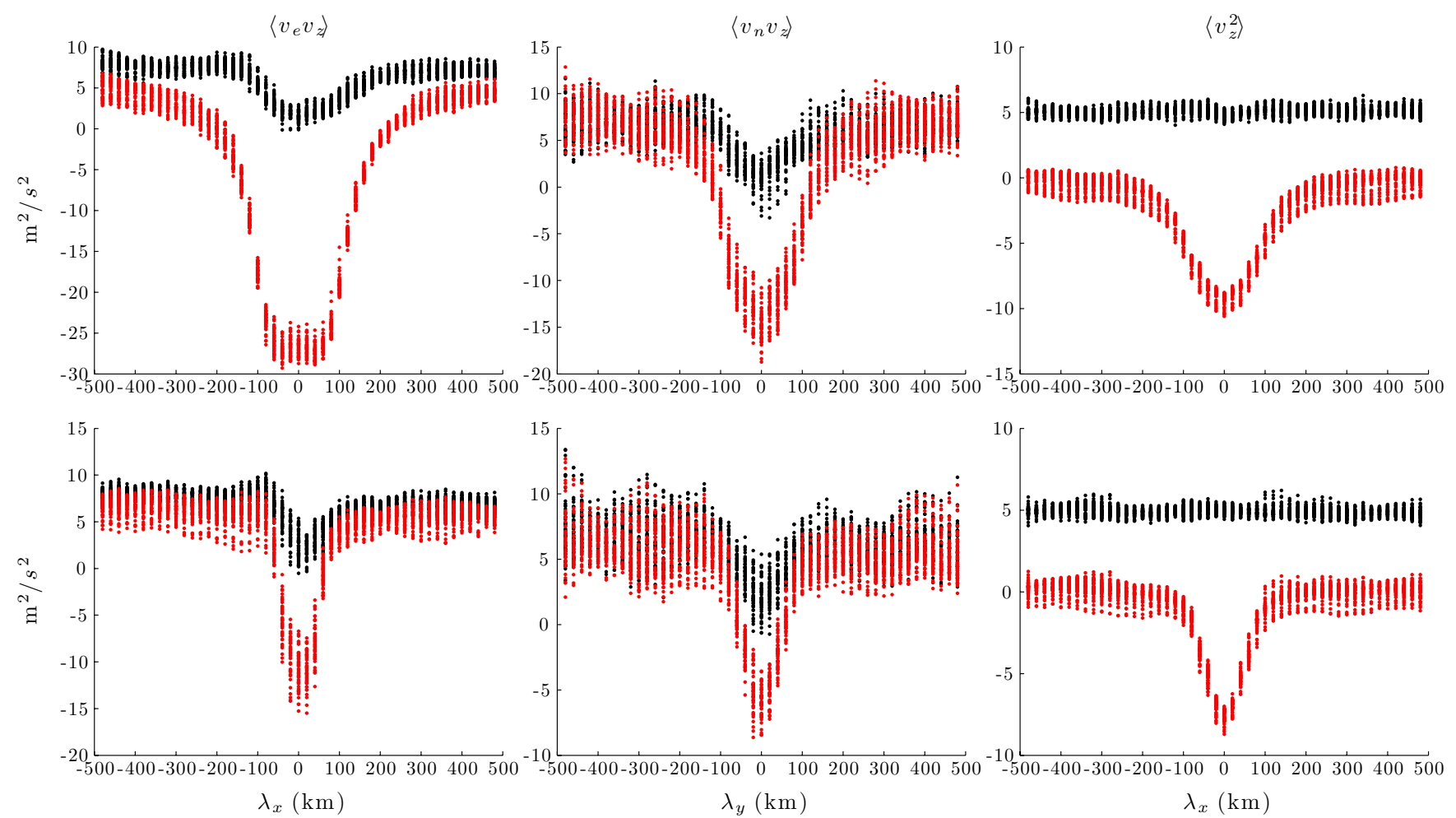

Fig. 6. Biases in the momentum fluxes and vertical velocity variance as a function of horizontal wavelength for beam map \#1 (see Table 1) (top row) and beam map \#2 (bottom row). Black curves show the solutions with only the variance terms included in the estimator, and red terms show results including the full covariance matrix. Simulations are with $\sigma_{e}=2 \mathrm{~m} \mathrm{~s}^{-1}$.

Franke, 1998). The right-hand panel shows the absolute error for this simulation, where it is quite clear for any given value of wind variances, $\sqrt{\left\langle v_{x}^{2}\right\rangle\left\langle v_{z}^{2}\right\rangle / N_{3}}$ (gray line) is a lower bound on the error (note that $v_{x}$ here is either component of the wind field, $v_{e}$ or $v_{n}$ ). The true error can be larger than this, depending on the magnitude of the correlation between the horizontal and vertical fluctuations. The importance of independent samples should be clear, as the only way to reduce the uncertainties on the momentum flux estimates is to incoherently average in time or space. Adding measurement errors (lower two rows of Fig. 5) clearly makes things worse, as one would expect. However, including the covariance terms significantly reduces the fractional and absolute errors $-\sim 30-40 \%$ in the $\sigma_{e}=2 \mathrm{~m} \mathrm{~s}^{-1}$ and $\sim 60-70 \%$ in the $\sigma_{e}=5 \mathrm{~m} \mathrm{~s}^{-1}$.

\subsection{Simulation 2: realistic wave field}

In this section, we extend the simulations to include a realistic wave field, mainly to investigate the effects of realistic horizontal variations in the perturbed radial velocity fields, similar to the approach taken by others (e.g., Fritts et al., 2010, 2012; Vincent et al., 2010). We simulate a case which is similar to Case 1 of Fritts et al. (2010) and Fritts et al. (2012), in which we have fixed mean, diurnal tide and semi- diurnal tidal amplitudes for the background winds in addition to imposed gravity wave variations.

In our modified Case 1, we have two gravity waves, with fixed amplitudes as given in Table 2, propagating orthogonally to one another with a horizontal wavelength and period drawn from a uniform random distribution from -500 to $500 \mathrm{~km}$, and 6 to $45 \mathrm{~min}$, respectively. These waves will lead to fixed wind variances and momentum fluxes, given in Table 2. For the simulation, we follow the following steps for $N_{1}$ iterations of the simulation to generate a realistic time series of measurements:

- Draw a wavelength and period for each of the two waves from a uniform random distribution, as described.

- For $N_{2}$ iterations, generate observed line-of-sight wind components using the background winds, horizontally varying gravity wave fields and measurement errors drawn from the zero-mean normal distribution with variance $\sigma_{e}^{2}$.

- Estimate the momentum flux and wind component variances for each of those iterations.

- Estimate the means and standard deviations of the variance and covariance terms over the $N_{2}$ iterations, which 

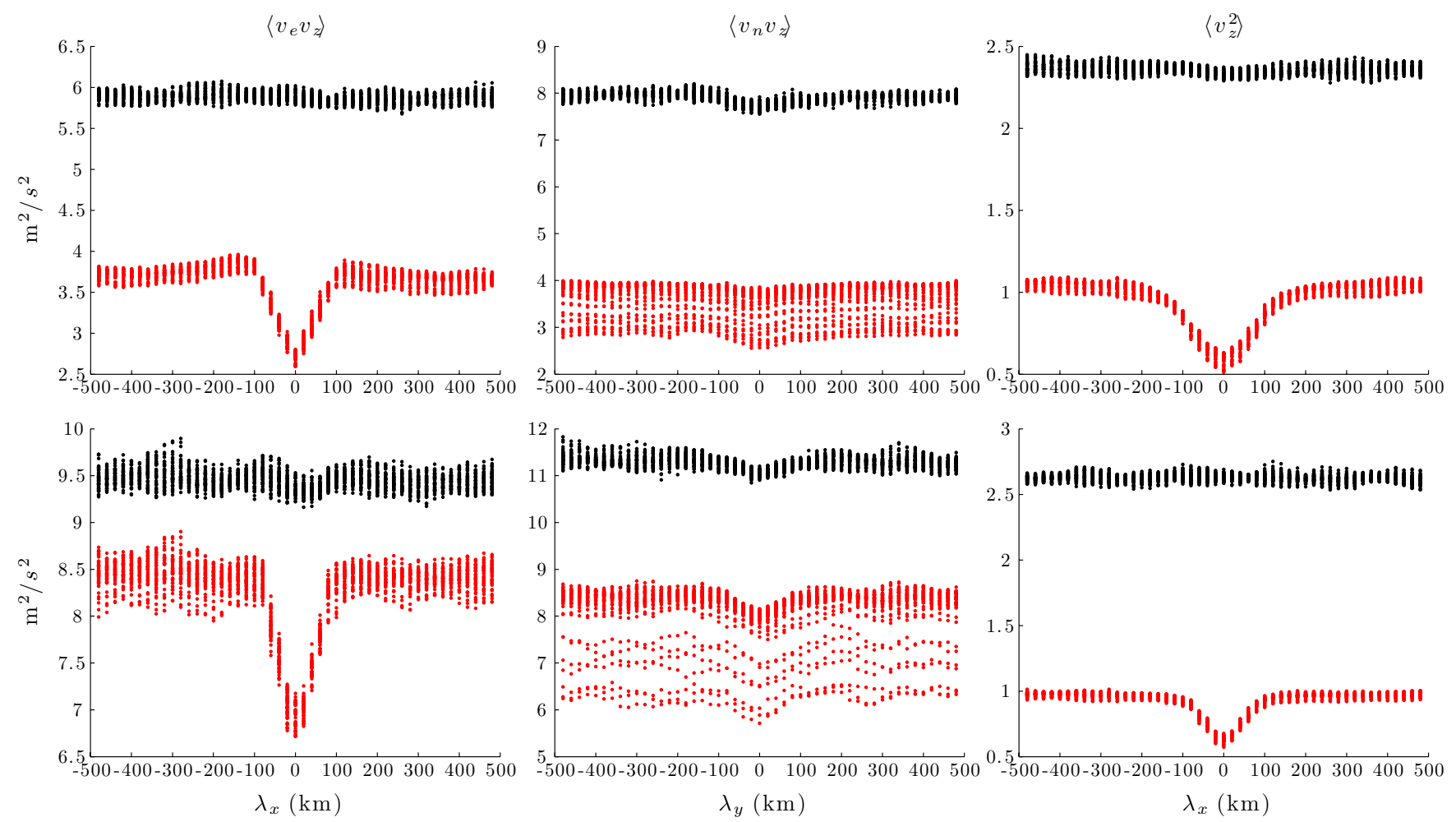

Fig. 7. Same as Fig. 6 for standard errors.

Table 2. Background winds, gravity wave parameters, and corresponding wind variances and momentum fluxes for the simulation in Sect. 3.3 .

\begin{tabular}{cccc}
\hline $\begin{array}{c}\text { Background winds } \\
\left(\mathrm{m} \mathrm{s}^{-1}\right)\end{array}$ & $\begin{array}{c}\mathrm{GW} 1 \\
\left(\mathrm{~m} \mathrm{~s}^{-1}, \mathrm{~km}, \mathrm{~min}\right)\end{array}$ & $\begin{array}{c}\mathrm{GW} 2 \\
\left(\mathrm{~m} \mathrm{~s}^{-1}, \mathrm{~km}, \mathrm{~min}\right)\end{array}$ & $\begin{array}{c}\text { Variances } \\
\left(\mathrm{m}^{2} \mathrm{~s}^{-2}\right)\end{array}$ \\
\hline$\left(U_{M}, V_{M}\right)=(20,10)$ & $\left(u^{\prime}, v^{\prime}, w^{\prime}\right)=(10,0,5)$ & $\left(u^{\prime}, v^{\prime}, w^{\prime}\right)=(0,20,2)$ & $\left(\left\langle v_{e}^{2}\right\rangle,\left\langle v_{n}^{2}\right\rangle,\left\langle v_{z}^{2}\right\rangle\right)=(50,200,14.5)$ \\
$\left(U_{D}, V_{D}\right)=(10,10)$ & $\lambda_{x}=-500$ to $500, \lambda y=\infty$ & $\lambda_{x}=\infty, \lambda y=-500$ to 500 & $\left\langle v_{e} v_{n}\right\rangle=0$ \\
$\left(U_{S D}, V_{S D}\right)=(50,50)$ & $\tau=6$ to 45 & $\tau=6$ to 45 & $\left(\left\langle v_{e} v_{z}\right\rangle,\left\langle v_{n} v_{z}\right\rangle\right)=(25,20)$ \\
\hline
\end{tabular}

can be used to compute the standard error on the estimate as well as any biases on the results.

The time series of the measurements corresponds to a twohour interval with samples every $3 \mathrm{~min}$, similar to the measurements that will be presented later. The simulation is performed at a single altitude of $80 \mathrm{~km}$.

It is crucially important to accurately estimate the covariance terms, for which a direct covariance calculation is not suitable because of scale-dependent propagation delays across the field-of-view. To do so, we employ a method wherein we take the Fourier transform of the crosscovariance function, or the cross-power spectral density, between measurement $i$ and measurement $j$. We high-pass filter the cross-spectrum in the frequency domain to eliminate low frequency motions with periods larger than an hour, which we are not able to address using the types of measurements targeted with this technique. We then integrate the cross-spectral density to obtain the magnitude of the covariance. The sign of the covariance is taken from the zero-lag of the cross-covariance function. Short-wavelength, spatially aliased waves may thus contribute to biased results, hence our desire to simulate a breadth of wave scales.

The results for Case 1 are shown in Figs. 6 and 7. Figure 6 shows the bias in the momentum fluxes and vertical velocity variance (average estimate minus true value) for the solutions including the variance terms in the estimator (black points) and the solutions including the covariance terms as outlined in this paper. Data are binned in terms of horizontal wavelength of the waves, since this was found to be a strong controlling factor on the nature of the bias. The top row of Fig. 6 utilises beam map \#1 as indicated in Table 1 . At large wavelengths, there is a clear bias in both methodologies, approaching $\sim 5-10 \mathrm{~m}^{2} \mathrm{~s}^{-2}$ in the momentum fluxes. At large horizontal wavelengths, the vertical velocity variance 

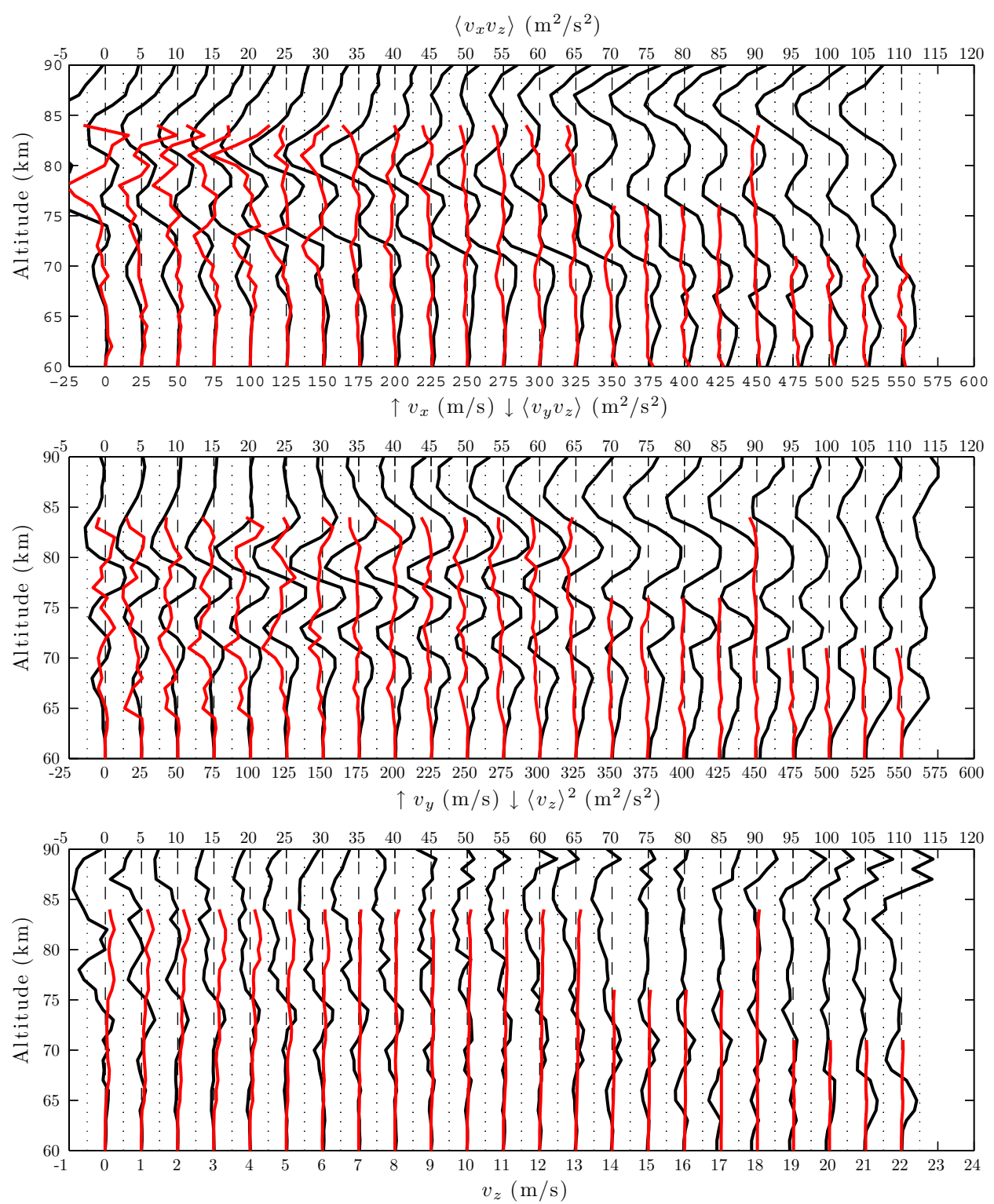

Fig. 8. Winds and momentum fluxes from 13:00-24:00 UT on 23 April 2008. Top: Zonal wind (black, $v_{x}$ ) and vertical flux of horizontal momentum (red, $\left\langle v_{e} v_{z}\right\rangle$, top axis). Winds are two-hour averages plotted every hour, separated by $25 \mathrm{~m} \mathrm{~s}^{-1}$. Momentum fluxes are computed over the two-hour period and are separated by $5 \mathrm{~m}^{2} \mathrm{~s}^{-2}$. Middle: Same for the meridional winds and momentum flux. Bottom: Vertical velocity (black, $v_{z}$ ) and vertical velocity variance (red, $\left\langle v_{z}\right\rangle^{2}$, top axis). Vertical winds are separated by $1 \mathrm{~m} \mathrm{~s}^{-1}$ and vertical velocity variances by $5 \mathrm{~m}^{2} \mathrm{~s}^{-2}$.

is unbiased as a result of accounting for the biased lineof-sight velocity variances. At small-horizontal wavelengths $(<\sim 100 \mathrm{~km})$ the method including the covariance terms becomes significantly oppositely biased. This is clearly an effect of the chosen geometry and scale-dependent propagation delays across the field-of-view. The bottom row of Fig. 6 shows the same results for beam map \#2, which has small off-zenith angles. Both the magnitude of the bias as well as the wavelength at which biases begins to deviate are significantly reduced. The importance of chosen beam geometry on the accuracy of the technique seems very clear. It should also be emphasized that the nature of any biases likely depends strongly on the wave field present at any given time.

Figure 7 shows a similar plot for the standard errors on the estimates and demonstrates a tradeoff between accuracy and precision. While eliminating the covariance terms from the approach results in a less-biased solution, standard errors are significantly reduced by retaining those terms. Similarly, choosing a beam pattern with smaller off-zenith angles increases the accuracy of the estimates, but also results in 

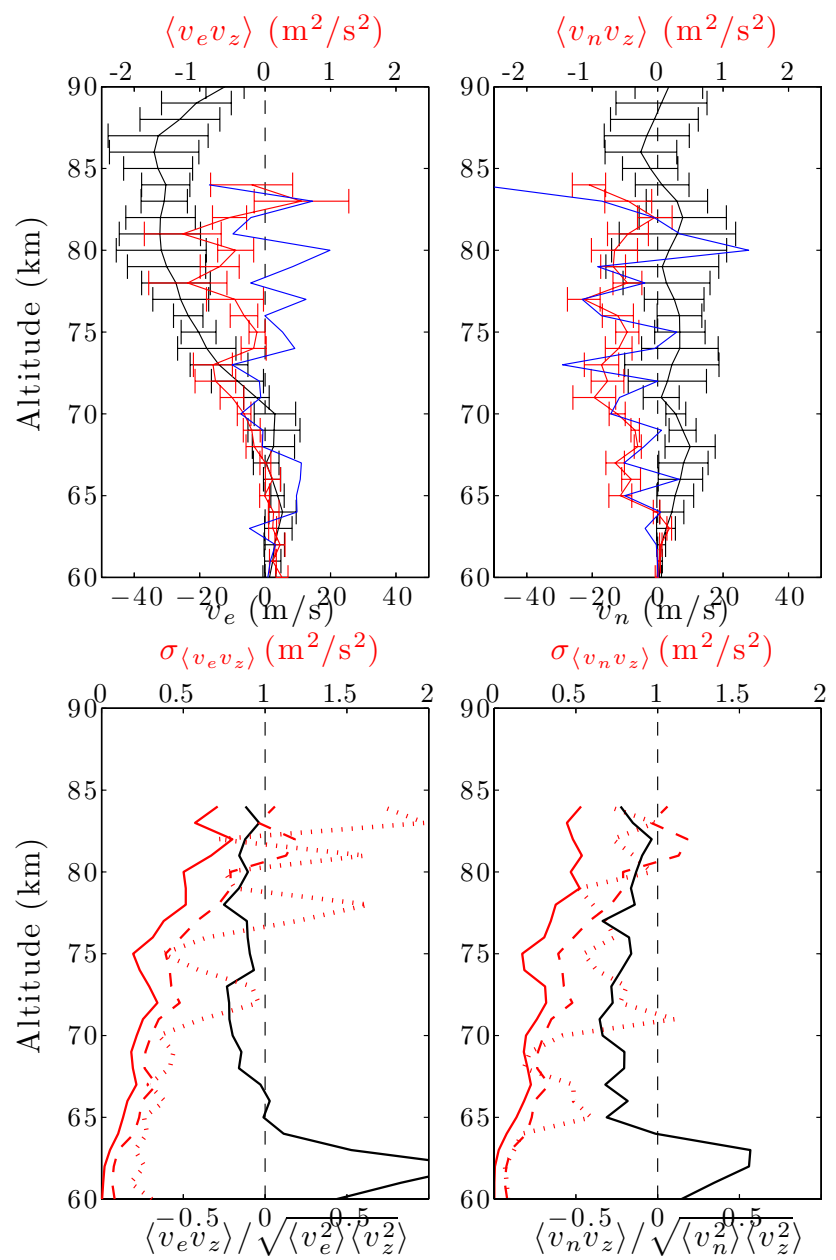

Fig. 9. Average momentum flux results from 13:00-24:00 UT on 23 April 2008. Top: Zonal (left) and meridional (right) wind (black lines) with error bars corresponding to the standard deviation of wind variation over the 11-h period. Red lines correspond to the averaged vertical flux of horizontal momentum in the zonal (left) and meridional (right) directions, with error bars corresponding to the standard error on the mean for the 11-h period. Blue lines correspond to the solution including only the variance terms. Bottom: Fraction of variance embodied in the momentum flux (black lines), averaged over the 11-h period, in the zonal (left) and meridional (right) directions. Red lines correspond to errors on the momentum flux estimates determined from the fitting algorithm averaged over the 11-h period (solid), average minimum error estimate using the geometric mean of the horizontal and vertical wind variances (dashed), and standard deviation of the data over the entire time period (dotted).

larger standard errors. In the cases where biases are smaller than expected errors (such as in the case of larger measurement errors), it would make sense to trade increased precision for accuracy. In any case, for a fixed experimental beam geometry, both solutions could be computed and compared to obtain the most quantitative picture possible.

\section{Results}

\subsection{Observations on 23 April 2008}

We have applied the technique described in this paper to PFISR data from 23 April 2008, which has been described and analysed in detail by Nicolls et al. (2010). A large- scale, large-amplitude inertia-gravity wave was observed over Poker Flat during this period. For our purposes here, we have averaged incoherent scatter radar spectra in $\sim 3 \mathrm{~min}$ integrations, corresponding to a sufficiently short average to capture the expected frequency range of velocity variability, and long enough to ensure that samples are approximately statistically independent. Spectra were fit as described in Nicolls et al. (2010) for spectral parameters including the line-of-sight drift velocity, taken to be equal to the component of the neutral wind along the radar line-of-sight. Lineof-sight velocities were averaged over $4 \mathrm{~km}$ in altitude (and analysed every $1 \mathrm{~km}$ ). The covariance matrix of the measurements were computed over a two-hour period, corresponding to 38 independent variance estimates (which can 

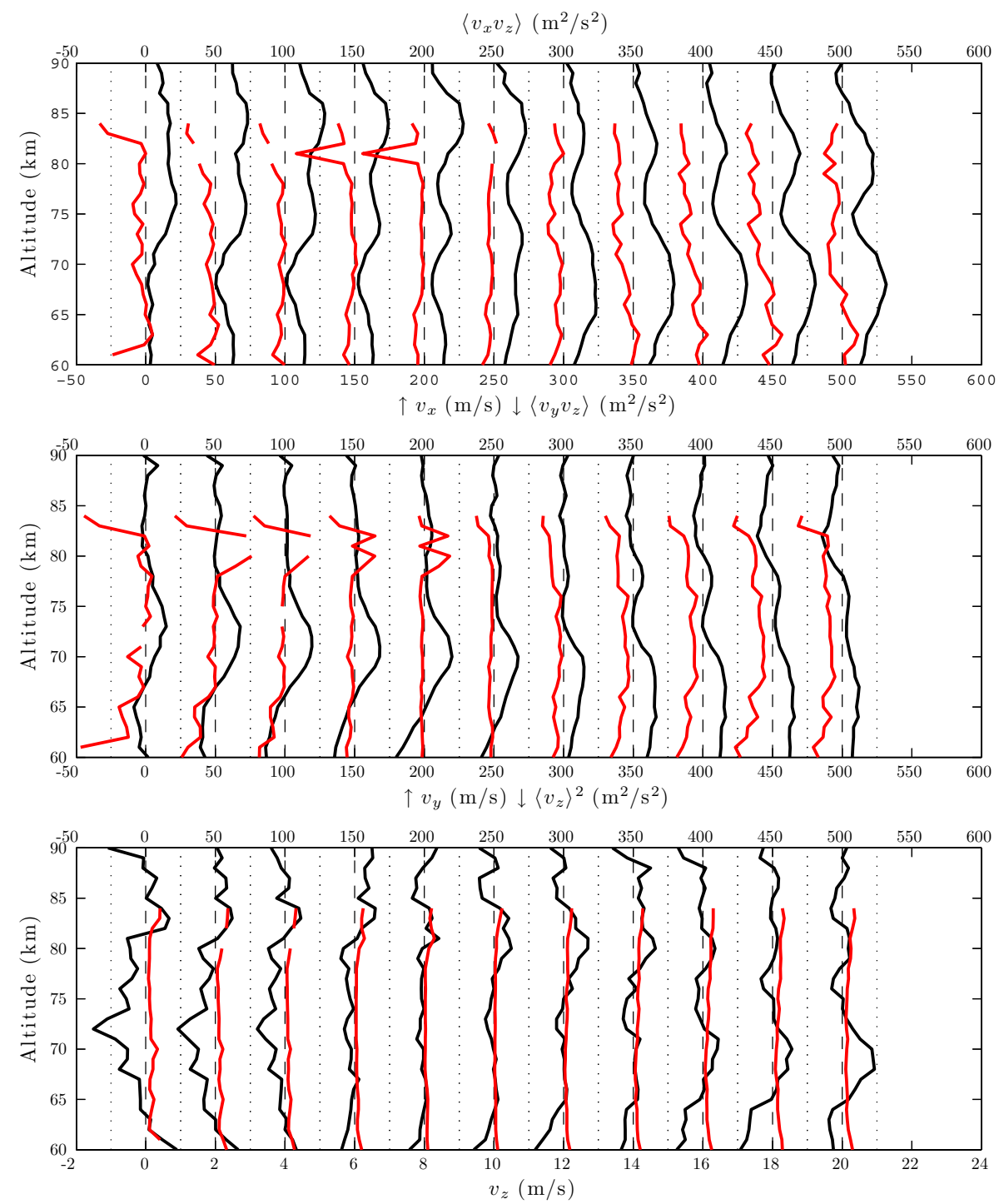

Fig. 10. Same as Fig. 8 for the period 18:00-24:00 UT on 21 December 2007. Note that momentum fluxes and vertical velocity variance spacing is $50 \mathrm{~m}^{2} \mathrm{~s}^{-2}$.

be equated to $N_{3}$ in the simulations in Sect. 3.2). As shown in the simulations and by Kudeki and Franke (1998), we expect the minimum error on the zonal and meridional momentum flux estimates over the two-hour periods to correspond to $\sqrt{\left\langle v_{e}^{2}\right\rangle\left\langle v_{z}^{2}\right\rangle / 38}$ and $\sqrt{\left\langle v_{n}^{2}\right\rangle\left\langle v_{z}^{2}\right\rangle / 38}$, respectively.

In Fig. 8, we show the two-hour averaged winds and momentum fluxes in the zonal (top) and meridional (middle) directions, plotted every hour. The short vertical wavelength wave activity is clearly evident in the zonal and meridional winds. The momentum flux is well-behaved and is roughly anti-correlated with the wave perturbations, increasing in amplitude between 75 and $85 \mathrm{~km}$. In the lower panel, we have plotted the averaged vertical velocities, along with the vertical velocity variances. The two-hour averaged vertical velocities fluctuate up to about $0.25 \mathrm{~m} \mathrm{~s}^{-1}$ (but more typically quite a bit less than that), and the variances for this dataset are quite small.

In Fig. 9, we show averaged results for the 11-h period from 13:00-24:00 UT on 23 April. The top panels of Fig. 9 show the averaged winds and momentum fluxes for this 11-h period, determined by averaging each 2-h estimate of the wind vector and momentum fluxes. Errorbars on the winds (black lines) correspond to standard deviations over the 11-h period and are meant to indicate the variability of the background winds embodied in Fig. 8. The winds are predominantly westward and northward over this period, increasing with altitude and peaking at an altitude of about $80 \mathrm{~km}$. The momentum fluxes are indicated by red lines, with errorbars corresponding to standard errors on the mean using the 2-h estimates over the 11-h period. The average momentum 

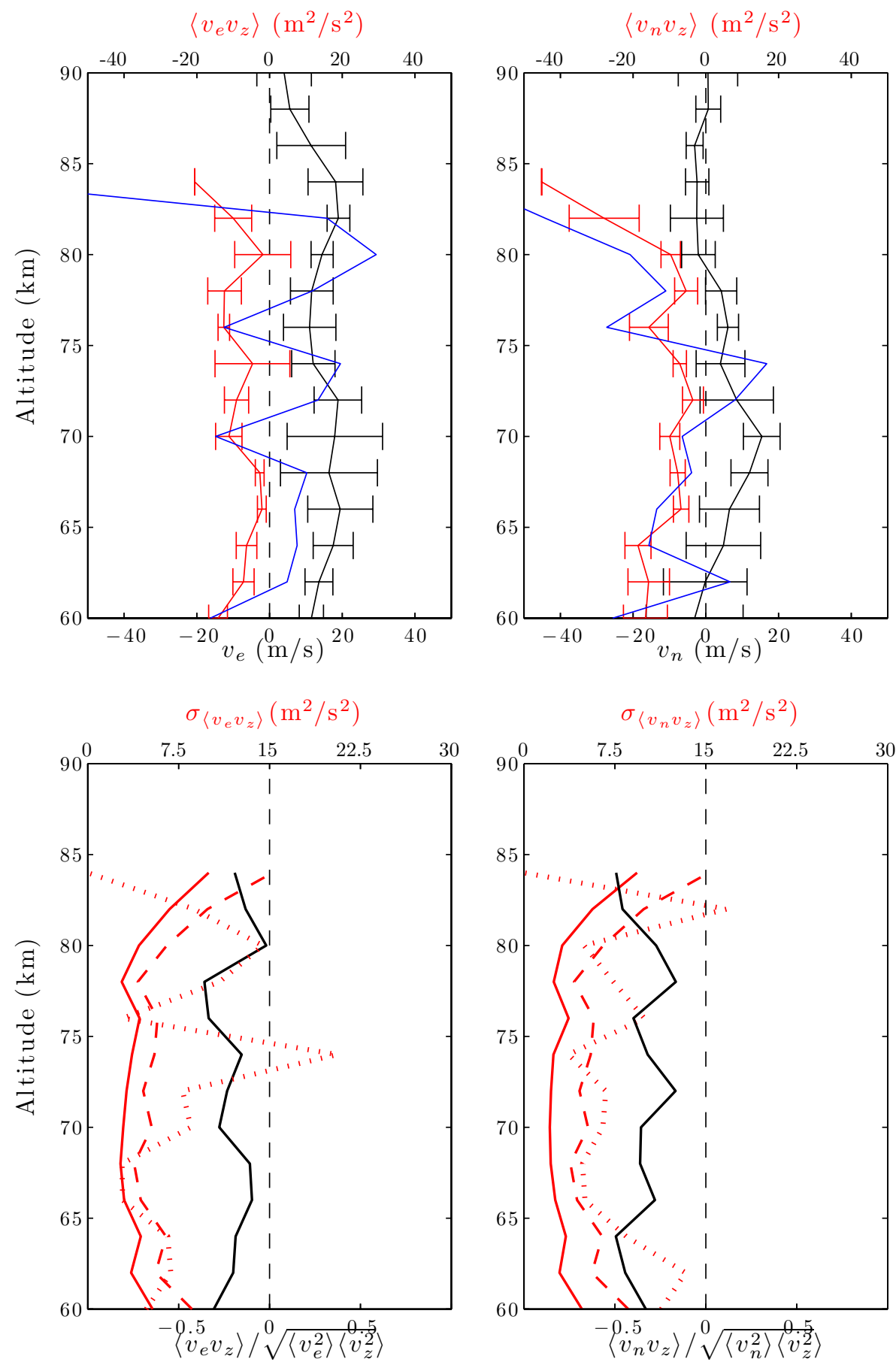

Fig. 11. Same as Fig. 9 for the period 18:00-24:00 UT on 21 December 2007.

fluxes are very small. The zonal flux is near zero except near $65-67 \mathrm{~km}$ and $75-80 \mathrm{~km}$ when it increases in the westward direction to an averaged value of $0.5-1 \mathrm{~m}^{2} \mathrm{~s}^{-2}$. The meridional flux is southward over most of the altitude range, with an averaged value of about -0.75 to $-1 \mathrm{~m}^{2} \mathrm{~s}^{-2}$. Blue curves show the solutions including only the variance terms and show higher variability (as expected), but rough agreement with the solutions including the covariance terms.

In the lower panels, we analyse the variability and expected errors of the measurements. The black lines show the correlation of the horizontal and vertical winds, which reach values of $\sim 40 \%$ of the geometric mean of the wind variances. Using the fact that there were at least 38 independent 
estimates of the variance in each $2-\mathrm{h}$ average, the dashed red lines show the expected (minimum) root-mean-square error on the momentum flux, again averaged over the 11-h period. The solid lines show the averaged errors determined from the mean-square error of the least-squares fitting process using the covariance matrix of the measurements. The dotted lines show the standard deviation of the measurements over the 13:00-24:00 UT period. The curves indicate expected errors on the 2-hr momentum flux estimates of $0-2 \mathrm{~m}^{2} \mathrm{~s}^{-2}$. The curves have very similar altitude trends, with the estimated errors from the covariance matrix of the measurements somewhat underestimating the variability of the data and the expected minimum errors.

\subsection{Observations on 21 December 2007}

In Figs. 10 and 11, we show results in the same format for 21 December 2007 for the period from 18:00-24:00 UT when signal-to-noise ratios were sufficient to determine winds and momentum fluxes. In this case, data were averaged for 2 min before fitting for the radar spectra, and the analysis was done with $6-\mathrm{km}$ altitude averaging (and computed every $3 \mathrm{~km}$ ) to ensure robust estimates. In this case, vertical velocity excursions were significantly larger than in the previous case, with vertical velocity variances approaching $\sim 10 \mathrm{~m}^{2} \mathrm{~s}^{-2}$ for individual two-hour windows, but more typically quite a bit less than that. The momentum fluxes are correspondingly considerably larger (peaking at $\sim 25 \mathrm{~m}^{2} \mathrm{~s}^{-2}$ ), with fluctuations that mimic the dynamic background wind variations. However, in this case, it is important to realise that the horizontal and vertical wind variations are sufficiently large that it may not be possible to robustly determine the momentum fluxes for a given two-hour window. As shown in the lower panels of Fig. 11, the expected averaged minimum root-mean-square error on the momentum flux is near $5 \mathrm{~m}^{2} \mathrm{~s}^{-2}$. The averaged results in Fig. 11 show that the average momentum flux for this $6-\mathrm{h}$ period is between $-10-0 \mathrm{~m}^{2} \mathrm{~s}^{-2}$ in the zonal direction and $\sim \pm 10 \mathrm{~m}^{2} \mathrm{~s}^{2}$ in the meridional direction (varying with altitude). These average results should be significant given the statistical uncertainties and demonstrate larger momentum fluxes on this day associated with the enhanced vertical velocities and variances. The predominant flux appears to be southwestward, opposite the direction of the background winds. Comparisons to the variance-only measurements show generally good agreement in the meridional direction, but indeterminate results in the zonal direction.

\section{Conclusions}

We have demonstrated a technique to estimate the covariance matrix of the wind field, including wind variances and momentum fluxes, using arbitrary, distributed measurements of line-of-sight winds. This approach is applicable especially to systems with flexible, but fixed pointing, such as phased array radars like the Poker Flat ISR, and is useful especially for estimates of higher-frequency and shorter lived wave inputs rather than long-term averages.

The approach is simply a generalization of previous techniques to include covariance terms. We demonstrated via simulation that the approach produces robust estimates of the wind variances and covariances that outperform the direct extension of the co-planar beam technique in the presence of finite measurement uncertainties because of the inclusion of covariance terms in the forward model. Accurate estimation of the covariance terms is crucial to the approach, and scaledependent propagation delays over the field-of-view of the system must be accounted for when estimating them. Misestimation of these terms and issues associated with experiment geometry, may lead to biased estimates of the momentum fluxes and wind variances. However, while complicating the approach, including the covariance terms provides a significant reduction in standard errors that may outweigh any potential biasing by misestimation. The covariance terms can easily be excluded to provide a less biased, but likely more error-prone, estimate of the momentum fluxes and wind variances.

Future work can optimize the beam positions, spatial averaging and time integration to take advantage of the expected range of momentum-containing scales. In addition, other sources of potential biases, such as beam-pointing accuracy, should be investigated.

Acknowledgements. PFISR data collection and analysis was supported by NSF cooperative agreement AGS-0608577. Research was supported by NSF grant AGS-0836142 to SRI International and NSF grant OPP-0839084 to CoRA.

Topical Editor C. Jacobi thanks two anonymous referees for their help in evaluating this paper.

\section{References}

Acott, P.: Mesospheric momentum flux studies over Fort Collins, CO, Ph.D. thesis, Colorado State University, 2009.

Antonita, T. M., Ramkumar, G., Kumar, K. K., and Deepa, V.: Meteor wind radar observations of gravity wave momentum fluxes and their forcing toward the Mesospheric Semiannual Oscillation, J. Geophys. Res., 113, D10115, doi:10.1029/2007JD009089, 2008.

Aster, R. C., Borchers, B., and Thurber, C. H.: Parameter Estimation and Inverse Problems, Elsevier, Inc., 2005.

Butler, T. W., Semeter, J., Heinselman, C. J., and Nicolls, M. J.: Imaging $F$ region drifts using monostatic phasedarray incoherent scatter radar, Radio Sci., 45, RS5013, doi:10.1029/2010RS004364, 2010.

Clemesha, B. R., Batista, P. P., Buriti da Costa, R. A., and Schuch, N.: Seasonal variations in gravity wave activity at three locations in Brazil, Ann. Geophys., 27, 1059-1065, doi:10.5194/angeo27-1059-2009, 2009. 
Fritts, D. C. and Vincent, R. A.: Mesospheric momentum flux studies at Adelaide, Australia - Observations and a gravity wave-tidal interaction model, J. Atmos. Sci., 44, 605-619, 1987.

Fritts, D. C. and Yuan, L.: Measurement of Momentum Fluxes near the Summer Mesopause at Poker Flat, Alaska., J. Atmos. Sci., 46, 2569-2579, 1989.

Fritts, D. C., Tsuda, T., Vanzandt, T. E., Smith, S. A., Sato, T., Fukao, S., and Kato, S.: Studies of Velocity Fluctuations in the Lower Atmosphere Using the MU Radar. Part II: Momentum Fluxes and Energy Densities., J. Atmos. Sci., 47, 51-66, 1990.

Fritts, D. C., Yuan, L., Hitchman, M. H., Coy, L., Kudeki, E., and Woodman, R. F.: Dynamics of the Equatorial Mesosphere Observed Using the Jicamarca MST Radar during June and August 1987, J. Atmos. Sci., 49, 2353-2371, 1992.

Fritts, D. C., Janches, D., Riggin, D. M., Sulzer, R. G. S. M. P., and Gonzalez, S.: Gravity waves and momentum fluxes in the mesosphere and lower thermosphere using $430 \mathrm{MHz}$ dualbeam measurements at Arecibo: 2. Frequency spectra, momentum fluxes, and variability, J. Geophys. Res., 111, D18108, doi:10.1029/2005JD006883, 2006.

Fritts, D. C., Janches, D., and Hocking, W. K.: Southern Argentina Agile Meteor Radar: Initial assessment of gravity wave momentum fluxes, J. Geophys. Res., 115, D19123, doi:10.1029/2010JD013891, 2010.

Fritts, D. C., Janches, D., Hocking, W. K., Mitchell, N. J., and Taylor, M. J.: Assessment of gravity wave momentum flux measurement capabilities by meteor radars having different transmitter power and antenna configurations, J. Geophys. Res., in press, 2012.

Fukao, S., Sato, T., Tsuda, T., Kato, S., and Inaba, M.: VHF Doppler radar determination of the momentum flux in the upper troposphere and lower stratosphere - Comparison between the threeand four-beam methods, J. Atmos. Ocean. Tech., 5, 57-69, 1988.

Heinselman, C. J. and Nicolls, M. J.: A Bayesian approach to electric field and E-region neutral wind estimation with the Poker Flat Advanced Modular Incoherent Scatter Radar, Radio Sci., 43, RS5013, doi:10.1029/2007RS003805, 2008.

Hitchman, M. H., Bywaters, K. W., Fritts, D. C., Coy, L., Kudeki, E., and Surucu, F.: Mean Winds and Momentum Fluxes over Jicamarca, Peru, during June and August 1987, J. Atmos. Sci., 49, 2372-2383, 1992.

Hocking, W. K.: A new approach to momentum flux determinations using SKiYMET meteor radars, Ann. Geophys., 23, 2433-2439, doi:10.5194/angeo-23-2433-2005, 2005.

Janches, D., Fritts, D. C., Sulzer, D. M. R., and Gonzalez, S.: Gravity waves and momentum fluxes in the mesosphere and lower thermosphere using $430 \mathrm{MHz}$ dual-beam measurements at Arecibo: 1. Measurements, methods, and gravity waves, J. Geophys. Res., 111, D18107, doi:10.1029/2005JD006882, 2006.

Kudeki, E. and Franke, S. J.: Statistics of momentum flux estimation, J. Atmos. Solar-Terr. Phys., 60, 1549-1553, 1998.

Lhermitte, R.: Doppler sonar observation of tidal flow, J. Geophys. Res., 88, 725-742, 1983.

Murayama, Y., Tsuda, T., and Fukao, S.: Seasonal variation of gravity wave activity in the lower atmosphere observed with the MU radar, J. Geophys. Res., 992, 23057-23070, doi:10.1029/94JD01717, 1994.

Murphy, D. J. and Vincent, R. A.: Estimates of momentum flux in the mesosphere and lower thermosphere over Adelaide, Aus- tralia, from March 1985 to February 1986, J. Geophys. Res., 981, 18617-18638, doi:10.1029/93JD01861, 1993.

Murphy, D. J. and Vincent, R. A.: Mesospheric momentum fluxes over Adelaide during the 2-day wave: Results and interpretation, J. Geophys. Res., 103, 28627, doi:10.1029/1998JD200001, 1998.

Nakamura, T., Tsuda, T., Yamamoto, M., Fukao, S., and Kato, S.: Characteristics of gravity waves in the mesosphere observed with the middle and upper atmosphere radar. I - Momentum flux, J. Geophys. Res., 98, 8899-8923, doi:10.1029/92JD02978, 1993.

Nicolls, M. J., Varney, R. H., Vadas, S. L., Stamus, P. A., Heinselman, C. J., Cosgrove, R. B., and Kelley, M. C.: Influence of an inertia-gravity wave on mesospheric dynamics: A case study with the Poker Flat Incoherent Scatter Radar, J. Geophys. Res., 115, D00N02, doi:10.1029/2010JD014042, 2010.

Placke, M., Hoffmann, P., Becker, E., Jacobi, C., Singer, W., and Rapp, M.: Gravity wave momentum fluxes in the MLT, Part II: Meteor radar investigations at high and midlatitudes in comparison with modeling studies, J. Atmos. Solar-Terr. Phys., 73, 911920, doi:10.1016/j.jastp.2010.05.007, 2011a.

Placke, M., Stober, G., and Jacobi, C.: Gravity wave momentum fluxes in the MLT, Part I: Seasonal variation at Collm $\left(51.3^{\circ} \mathrm{N}, 13.0^{\circ} \mathrm{E}\right)$, J. Atmos. Solar-Terr. Phys., 73, 904-910, doi:10.1016/j.jastp.2010.07.012, 2011 b.

Reid, I. M. and Vincent, R. A.: Measurements of mesospheric gravity wave momentum fluxes and mean flow accelerations at Adelaide, Australia, J. Atmos. Terr. Phys., 49, 443-460, 1987.

Reid, I. M., Ruester, R., Czechowsky, P., and Schmidt, G.: VHF radar measurements of momentum flux in the summer polar mesosphere over Andenes (69 deg N, 16 deg E), Norway, Geophys. Res. Lett., 15, 1263, doi:10.1029/GL015i011p01263, 1988.

Sato, K.: Vertical wind disturbances in the troposphere and lower stratosphere observed by the MU radar, J. Atmos. Sci., 47, $2803-$ 2817, 1990.

Sato, K.: Small-Scale Wind Disturbances Observed by the MU Radar during the Passage of Typhoon Kelly., J. Atmos. Sci., 50, 518-537, 1993.

Sato, K.: A statistical study of the structure, saturation and sources of inertio-gravity waves in the lower stratosphere observed with the MU radar, J. Atmos. Terr. Phys., 56, 755-774, 1994.

Tarantola, A.: Inverse problem theory and methods for model parameter estimation, Society for Industrial and Applied Mathematics, 2005.

Thorsen, D., Franke, S. J., and Kudeki, E.: A new approach to MF radar interferometry for estimating mean winds and momentum flux, Radio Sci., 32, 707-726, 1997.

Thorsen, D., Franke, S. J., and Kudeki, E.: Statistics of momentum flux estimation using the dual coplanar beam technique, Geophys. Res. Lett., 27, 3193-3196, doi:10.1029/1999GL011196, 2000.

Tsuda, T., Murayama, Y., Yamamoto, M., Kato, S., and Fukao, S.: Seasonal variation of momentum flux in the mesosphere observed with the MU radar, Geophys. Res. Lett., 17, 725-728, 1990.

Vincent, R. A. and Reid, I. M.: HF Doppler measurements of mesospheric gravity wave momentum fluxes, J. Atmos. Sci., 40, 13211333, 1983. 
Vincent, R. A., Kovalam, S., Reid, I. M., and Younger, J. P.: Gravity wave flux retrievals using meteor radars, Geophys. Res. Lett., 37, L14802, doi:10.1029/2010GL044086, 2010.

Wang, D.-Y. and Fritts, D. C.: Mesospheric Momentum Fluxes Observed by the MST Radar at Poker Flat, Alaska., J. Atmos. Sci.,
47, 1512-1521, 1990.

Wang, D.-Y. and Fritts, D. C.: Evidence of Gravity Wave-Tidal Interaction Observed near the Summer Mesopause at Poker Flat, Alaska., J. Atmos. Sci., 48, 572-583, 1991. 